\title{
Impact of the environment and the topology on the performance of hierarchical body area networks
}

\author{
Jean-Michel Dricot ${ }^{1 *}$, Stéphane Van Roy ${ }^{1}$, Gianluigi Ferrari ${ }^{2}$, François Horlin ${ }^{1}$ and Philippe De Doncker ${ }^{1}$
}

\begin{abstract}
Personal area networks and, more specifically, body area networks (BANs) are key building blocks of future generation networks and of the Internet of Things as well. In this article, we present a novel analytical framework for network performance analysis of body sensor networks with hierarchical (tree) topologies. This framework takes into account the specificities of the on-body channel modeling and the impact of the surrounding environment. The obtained results clearly highlight the differences between indoor and outdoor scenarios, and provide several insights on BAN design and analysis. In particular, it will be shown that the BAN topology should be selected according to the foreseen medical application and the deployment environment.
\end{abstract}

\section{Introduction}

Recent advances in ultra-low power sensors have fostered the research in the field of body-centric networks, also referred to as body area networks (BANs) [1-4]. In these networks, a set of nodes (called sensors) is deployed on the human body. They aim at monitoring and reporting several physiological values, such as blood pressure, breath rate, skin temperature, or heart beating rate. Most of the time, sensing is performed at low rates but, in emergency situations, the network load may increase in seconds. Therefore, an in-depth analysis of the network outage, throughput, and achievable transmission rate can give insights on the maximum supported reporting rate and the corresponding performance.

In $[5,6]$, we have considered a preliminary link-level performance analysis of BANs with centralized topologies. In the current study, we extend this approach, integrating the propagation channel characteristics and the impact of the hierarchy in a general network-level performance analysis framework. All considered networks will have hierarchical topologies, i.e., the sensor nodes will not be directly connected to a central controller. The modeling of the BAN channel has recently been thoroughly investigated [7-11]. The main findings on the body radio propagation channel can be summarized as follows. First, the average value of the power decreases as

\footnotetext{
* Correspondence: jdricot@ulb.ac.be

'OPERA-Wireless Communications Group, Université Libre de Bruxelles, Belgium

Full list of author information is available at the end of the article
}

an exponential function of the distance. However, unlike classical propagation models, where the received power is a decreasing function of the distance of the form $d^{-\alpha}$, the authors of $[12,13]$ show that a law of the form $10^{\gamma d}(\gamma<0)$ characterizes more accurately body radio propagation. Second, the propagation channel is subject to distinct propagations mechanisms with respect to the location of the sensors on the body. More precisely, on-body propagation and reflections from the environment act jointly to create a particular propagation mechanism that is specific to BANs.

This article addresses the development of a specific framework for the accurate evaluation of the impact of the body-specific propagation and network topology. Our results are derived by means of the link throughput analysis, this metric being a traditional measure of how much traffic can be delivered, per time unit, by the network $[14,15]$. Therefore, our analysis is expedient to understand the level of information which could be collected and processed in body-related applications (e.g., health or fitness monitoring). Furthermore, since energy is critical in the design of autonomous BANs in the context of medical applications [16-18], an accurate evaluation of the impacts of the BAN topology and transmission rate on the energy consumption is of fundamental interest.

The slotted ALOHA multiple access scheme [19] was recently proposed by the IEEE 802.15 .6 working group as one of the reference medium access control (MAC) schemes for the wireless body networks in the context of the narrowband communications [20]. In particular, in 
each time slot, the nodes are assumed to transmit independently with a certain fixed probability [21]. This approach is supported by the observations in [[22], p. $278]$ and $[21,23]$, where it is shown that the traffic generated by nodes using a slotted random access MAC protocol can be modeled by means of a Bernoulli distribution. In fact, in more sophisticated MAC schemes, the probability of transmission at a node can be modeled as a function of general parameters, such as queuing statistics, the queue-dropping rate, the channel outage probability incurred by fading [24], the adaptation of the sampling to rate to patient's condition [25], the MAC strategy used [26], etc. Since the impact of these parameters is not the focus of the this study, the interested reader is referred to the existing literature [27-29] for further details.

The principal contributions of this article can be summarized as follows. First, a comprehensive and detailed analytic framework for BAN performance evaluation is developed, obtaining closed-form expressions for the link probabilities of outage in the context of multi-user communications. This framework encompasses the effect of the environment, the topology, and the traffic intensity. Next, different topologies, corresponding to various medical applications, are characterized in terms of achievable throughput. Finally, the performance of each topology is discussed, and practical insights are given on how to instantiate a real-life BAN with respect to the application demands and propagation context. Furthermore, throughout this entire article the indoor and the outdoor environments are treated separately and properly compared.

The remainder of this article is organized as follows. In Section 2, the propagation mechanisms are introduced and characterized. In Section 3, the conditional success probability of a link transmission for a node, given the transmitter-receiver and interferer-receiver distances, is derived. In the same section, the minimum required transmit power, over a given link, in the absence of any interfering node is computed in both indoor and outdoor environments. Then, in Section 4, the tree topologies analyzed in this article are presented, and the traffic model is discussed. Finally, in Section 5, an extensive performance analysis, in terms of network throughput and energy consumption, is performed. Section 6 concludes this article.

\section{Propagation mechanisms}

In order to build an accurate model for the on-body propagation, a Rohde \& Schwartz ZVA-24 vector network analyzer was employed to capture the complex-valued frequency-domain transfer functions between 3 and $7 \mathrm{GHz}$, with a frequency step of $50 \mathrm{MHz}$. Omnidirectional Skycross SMT-3T010M ultra-wide band antennas were used during the entire measurement campaign. Their small-size $(13.6 \mathrm{~mm} \times 16 \mathrm{~mm} \times 3 \mathrm{~mm})$ and low profile characteristics precisely match the body sensor requirements. These antennas were separated from the body skin by about 5 $\mathrm{mm}$ to ensure a return loss value $S_{11} \leq-9 \mathrm{~dB}$. Finally, lowloss and phase-stable cables interconnect all components, and the IF-bandwidth was set to $100 \mathrm{~Hz}$ to enlarge the dynamic range to about $120 \mathrm{~dB}$.

The experimental scenario is presented in Figure 1 and can be described as follows. The measurements were carried out around the $94 \mathrm{~cm}$ of the waist of a man $(1 \mathrm{~m} 87,83 \mathrm{~kg})$ whose body is in a standing position, arms hanging along the side. The transmit antenna is placed around the body at a distance $d$ from the receive antenna, which is located at the middle axis of the torso.

\section{A. Measurements}

First, the diffraction mechanism is analyzed by gradually shifting the transmitter around the body. The spatial values of the power are extracted from seven different sites separated by $4 \mathrm{~cm}$ each. For each level, the transmitter is also shifted one level below and one level above, and the observed measures are averaged. Second, the impact of the reflections off the surrounding environment was investigated for five positions of the transmitter around the body. Repeated measures are taken by positioning the human body on a rectangular grid of $7 \times 7$ position, each separated by $4 \mathrm{~cm}$. This procedure is performed for a set of 20 locations in a standard office room with a surface of about $20 \mathrm{~m}^{2}$.

The baseband frequency response at the receiver was then converted into the delay domain using an inverse discrete Fourier transform [30]. Next, a Hamming window was applied to reduce the side lobes up to $-43 \mathrm{~dB}$ for the second lobe. The resulting complex impulse response allows a description of the BAN channel with a delay resolution of up to 0.25 ns. As shown in Figures 2 and 3, the different multipath and scattering mechanisms are well distinguished as a function of time. More precisely, the diffraction around the body is followed by the reflections off the environment. Both propagation mechanisms can be efficiently separated by applying a rectangular time gating at $7 \mathrm{~ns}$. Finally, the narrowband power of the

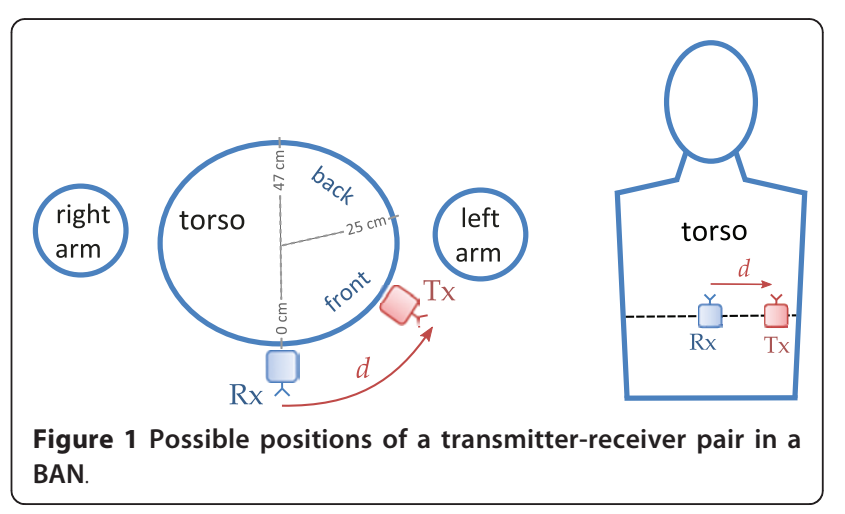




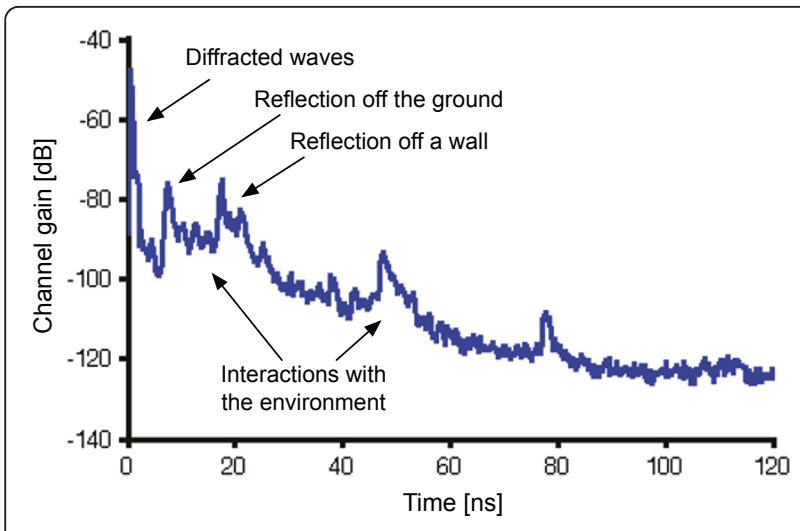

Figure 2 Power delay profile as a function of the time in an indoor environment and for $d \leq 25 \mathrm{~cm}$ (body front).

two distinct contributions mechanism is estimated by integrating the complex values of the temporal taps over each sub-channel.

The conclusions of this extensive measurement campaign, also highlighted in [13], can be summarized in three points. Firstly, there is propagation through the body. However, when high transmission frequencies are considered, the attenuation undergone by these waves is relevant and the corresponding contribution can be neglected.

A second mechanism corresponds to guided diffraction around the body. This mechanism is consistent with a surface wave propagation, and its properties depend on the body specific characteristics.

Finally, the last propagation contribution comes from the surrounding environment. More precisely, the third propagation mechanism originates from reflections off the body limbs (arms and legs) and the surrounding objects (walls, floor, and ceiling). Obviously, this mechanism is observed only in an indoor environment.

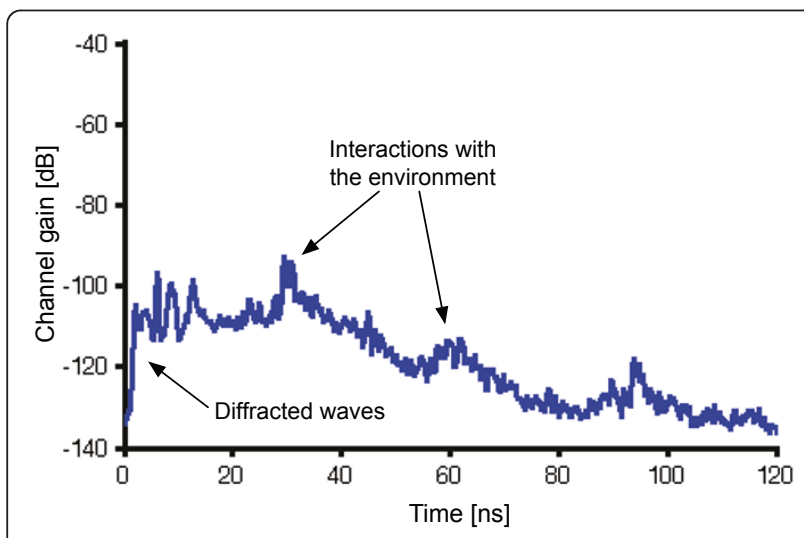

Figure 3 Power delay profile as a function of the time in an indoor environment and for $d>25 \mathrm{~cm}$ (body back).
Based on an extensive measurement campaign, we now present accurate statistical models corresponding to the propagation mechanisms described above.

\section{B. On-body propagation (guided diffraction)}

As previously emphasized in [12,31], the average received power (in $\mathrm{dB}$ scale) is the following linearly decreasing function of the distance:

$$
\mathbb{E}[\mathbf{P}(d)]=P+L_{\mathrm{ref}}+10 \gamma\left(d-d_{\mathrm{ref}}\right), \quad d \geq d_{\mathrm{ref}}
$$

where $\mathbf{P}(d)$ is the instantaneous received power (dimension: $[\mathrm{W}]$ ) at distance $d$ (dimension: $[\mathrm{m}]), P$ is the transmit power (dimension: [W]), $d_{\text {ref }}$ is a reference distance (dimension: $[\mathrm{m}]), L_{\mathrm{ref}}$ is the gain at the reference distance (adimensional, in $\mathrm{dB}$ ), and $\gamma$ is a suitable constant (dimension: $\left[\mathrm{m}^{-1}\right]$ ). For instance, typical experimental values for these parameters are $d_{\text {ref }}=8 \mathrm{~cm}, L_{\text {ref }}$ $=-57.42 \mathrm{~dB}$, and $\gamma=-124 \mathrm{~dB} / \mathrm{m}$ [31].

The average received power, in linear scale, can then be expressed as follows:

$$
\mathbb{E}[\mathbf{P}(d)]=P \cdot L(d), \quad d \geq d_{\mathrm{ref}}
$$

where

$$
\begin{aligned}
L(d) & =\underbrace{10^{\left(L_{\mathrm{ref}}-10 \gamma d_{\mathrm{ref}} / 10\right.}}_{\triangleq L_{0}} \times 10^{\gamma d} \\
& =L_{0} 10^{\gamma d}, \quad d \geq d_{\mathrm{ref}},
\end{aligned}
$$

where $L_{0}$ is a function of $L_{\text {ref }}, d_{\text {ref }}$, and $\gamma^{\text {a }}$ In Figure 4a, the loss $L$ is shown as a function of the distance, considering narrowband transmissions at $5 \mathrm{GHz}$. More precisely, in Figure 4a, experimental measurements (circles) and their linear interpolation (solid line) are shown. Finally, using (3) in (2) one obtains

$$
\mathbb{E}[\mathbf{P}(d)]=P L_{0} 10^{\gamma d} .
$$

While expression (4) characterizes the average value, it does not provide insights on the instantaneous distribution of the received power. In [31], it has been experimentally observed that the on-body propagation channel is characterized by slow large-scale fading (i.e., shadowing). More precisely, the instantaneous received power at distance $d$ can be expressed as follows:

$$
\mathbf{P}(d)=P L_{0} 10^{\gamma d} \mathbf{X}
$$

where $\mathbf{X}$ is a random variable (RV) which depends on the channel characteristics. As shown in [32] and confirmed by our measurements, $\mathbf{X}$ has a log-normal distribution $^{\mathrm{b}}$ with parameters $\mu$ and $\sigma$, where $\sigma_{\mathrm{dB}}$ typically ranges from 4 to $10 \mathrm{~dB}, \mu_{\mathrm{dB}}$ is the average path loss on the link (dimension: $[d B]$ ). Since the loss is accounted for by the term $L(d)$, it follows that $\mu_{\mathrm{dB}}=0 \mathrm{~dB}$, and the 


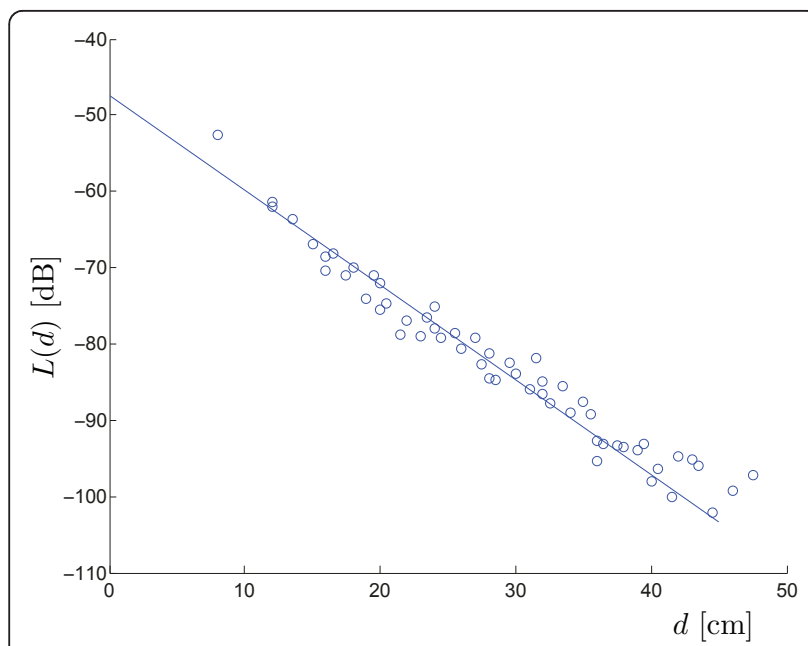

(a)

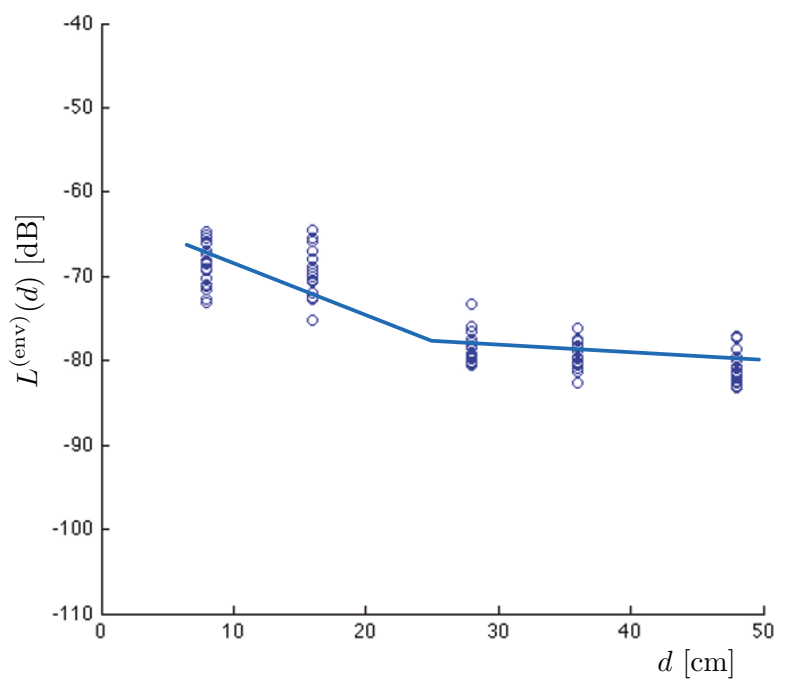

(b)

Figure 4 Propagation loss as a function of the distance: (a) onbody propagation, and (b) propagation through reflections off the environment. In both cases, experimental results (circles) and their linear (or piece-wise linear, in case (b)) interpolations (solid line) are shown.

cumulative distribution function (cdf) of $\mathbf{X}$ reduces to the following:

$$
F_{\mathrm{X}}(x ; 0, \sigma)=\frac{1}{2}-\frac{1}{2} \operatorname{erf}\left(\frac{-10 \log _{10} x}{\sigma \sqrt{2}}\right)
$$

with the following corresponding probability density function:

$$
f_{\mathrm{X}}(x ; 0, \sigma)=\frac{10}{(\ln 10) x \sqrt{2 \pi} \sigma} \exp \left\{-\frac{\left(10 \log _{10} x\right)^{2}}{2 \sigma^{2}}\right\} \text {. }
$$

\section{Reflections off the environment}

The second significant propagation mechanism originates from the multiple reflections off the environment. A substantial measurement campaign has shown that the contribution of the environment can be considered, on average, as an additive, constant power when the transmission distance is significant (i.e., when $d>25$ $\mathrm{cm})$. The obtained results are shown in Figure $4 \mathrm{~b}$, and the power received by means of reflections from the surrounding environment is shown as a function of the distance. It can be observed that when $d>25 \mathrm{~cm}$, the value of the loss is, on average, around $-78 \mathrm{~dB}$. More precisely, for $d>25 \mathrm{~cm}$, the average value of the received power can be expressed in logarithmic scale as follows:

$$
\mathbb{E}\left[\mathbf{P}_{\mathrm{env}}\right]=P_{\mathrm{env}} \triangleq P+L_{\mathrm{dB}}^{(\text {env })},
$$

where $P$ is the transmit power and $L_{\mathrm{dB}}^{(\mathrm{env})} \simeq-78 \mathrm{~dB}$. Alternatively, the average received power can be expressed in linear scale as

$$
\mathbb{E}\left[\mathbf{P}_{\mathrm{env}}\right]=P_{\mathrm{env}} \triangleq P \cdot L^{(\mathrm{env})},
$$

where $L^{(\text {env })}=10^{L_{\mathrm{dB}}^{(\mathrm{w})} / 10 \text {. }}$

Our measurement campaign has shown that the propagation channel can be accurately characterized as narrowband Rayleigh block fading. Therefore, the instantaneous received power $\mathbf{P}_{\mathbf{e n v}}$ has the following exponential distribution [33]:

$$
f_{\mathrm{P}_{\mathrm{env}}}(x)=\frac{1}{P_{\mathrm{env}}} \exp \left\{-\frac{x}{P_{\mathrm{env}}}\right\} .
$$

\section{A unified BAN propagation model}

The combination of the two propagation mechanisms presented in Sections 2-B and 2-C allows to derive a unified propagation model for a generic BAN. It can be observed that the degree of importance of each mechanism depends on the distance between transmitter and receiver. More precisely, in close proximity, the dominant propagation mechanism is the on-body propagation described in Section 2-B. Above the cross-over distance $d_{\text {cross }} \approx 25 \mathrm{~cm}$, the contribution of the environment becomes dominant, and the second propagation mechanism, presented in Section 2-C, is the only relevant one.

Therefore, a unified propagation model can be characterized as follows:

- in an outdoor environment, the average received power can be computed using (4) (i.e., $\left.\mathbb{E}[\mathbf{P}(d)] \propto P 10^{\gamma d}\right)$ and the instantaneous received 
power is determined by the log-normal fading channel model given by (5);

- in an indoor environment,

- if $d \leq d_{\text {cross }}$, the average received power can be computed using (4) (i.e., $\mathbb{E}[\mathbf{P}(d)] \propto P 10^{\gamma d}$ ) and the log-normal fading in (5) is used;

- if $d>d_{\text {cross }}$, the average received power is approximately constant (i.e., $\mathbb{E}[\mathbf{P}(d)]=P L^{(\text {env) }}$ ) and the instantaneous received power, owing to a Rayleigh faded channel model, has the distribution given by (8).

In Figure 5, the average path loss is shown as a function of the distance. In particular, the overall (unified) path loss can be expressed as follows:

$$
\begin{aligned}
L^{(\text {indoor })}(d) & =\max \left\{L_{0} 10^{\gamma d}, L^{(\text {env) }}\right\}, \\
L^{\text {(outdoor) }}(d) & =L_{0} 10^{\gamma d} .
\end{aligned}
$$

\section{Link-level performance analysis}

In this article, we consider multi-user communicationsin a BAN, all sensors need to transmit to a central controller and, in this sense, the scenario at hand can be interpreted as a multi-user scenario. The transmission over a link of interest is denoted with the subscript " $0 . "$ Besides the intended transmitter, other nodes may be interfering. Depending on their distance to the receiver, the interfering nodes will be denoted differently. More precisely,

- in an indoor scenario, the interferers located at distances shorter than $d_{\text {cross }}$ are referred to as "closerange interferers," their number is indicated as

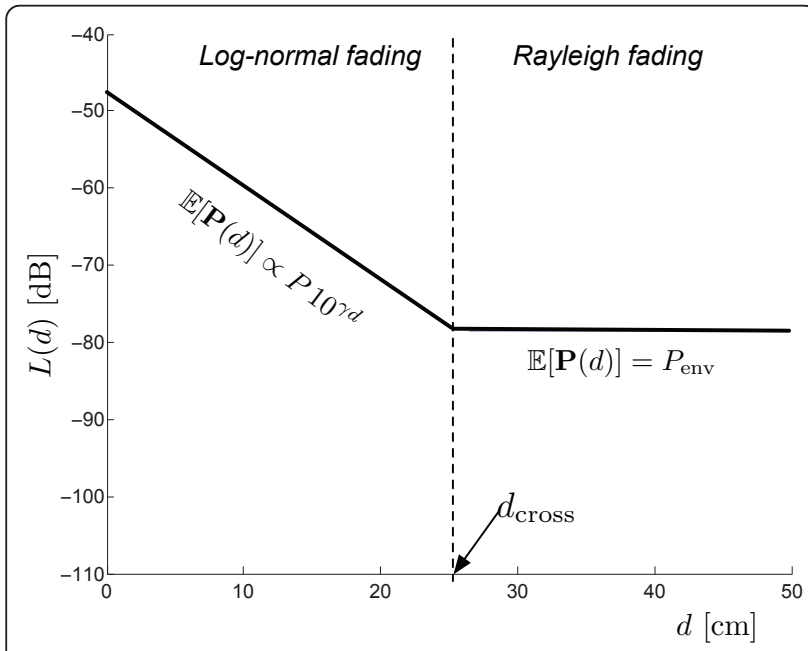

Figure 5 Generic propagation model (on-body and environment reflection superimposed)
$N_{\text {close }}$, and the generic node will be denoted with a subscript $i \in \mathcal{N}_{\text {close }} \triangleq\left\{1,2, \ldots, N_{\text {close }}\right\}$;

- in an indoor scenario, the interferers located at distances longer than $d_{\text {cross }}$ are referred to as "far-range interferers," their number is indicated as $N_{\text {far }}$, and the generic node will be denoted with a subscript $j \in \mathcal{N}_{\text {far }} \triangleq\left\{1,2, \ldots, N_{\text {far }}\right\}$;

- in an outdoor scenario, the number of interferers is indicated as $N_{\text {out }}$, and the generic node will be denoted with a subscript $k \in \mathcal{N}_{\text {out }} \triangleq\left\{1,2, \ldots, N_{\text {out }}\right\}$.

The transmission state of the a node at time $t$ is characterized by the following indicator variable:

$$
\boldsymbol{\Lambda}(t)=\left\{\begin{array}{l}
1 \text { if the node is transmitting at time } t \\
0 \text { if the node is silent at time } t
\end{array}\right.
$$

Assuming slotted transmissions (i.e., $t$ can assume multiples of the slot time), a simple random access scheme is such that, at each time slot, a node transmits with probability $q$ [[34], p. 278]. Therefore, $\left\{\boldsymbol{\Lambda}_{i}(t)\right\}_{t=1}^{\infty}$, $\left\{\boldsymbol{\Lambda}_{j}(t)\right\}_{t=1}^{\infty},\left\{\boldsymbol{\Lambda}_{j}(t)\right\}_{t=1}^{\infty}, j \in \mathcal{N}_{\text {far }}$, and $\left\{\boldsymbol{\Lambda}_{k}(t)\right\}_{t=1}^{\infty}, k \in \mathcal{N}_{\text {out }}$ are sequences of Bernoulli RVs with $\mathbb{P}\left\{\Lambda_{i}(t)=1\right\}=\mathbb{P}\left\{\Lambda_{j}(t)=1\right\}=q, \forall t, i, j, k$.

A transmission in a given link is successful if and only if the signal-to-noise and interference ratio (SINR) at the receiver is above a certain threshold $\theta$. This threshold value depends on the receiver characteristics, the modulation format, and the coding scheme, among other aspects. The SINR at the receiving node of the link is given by

$$
\operatorname{SINR} \triangleq \frac{\mathbf{P}_{0}\left(d_{0}\right)}{N_{0} B+\mathbf{P}_{\text {int }}}
$$

where $\mathbf{P}_{0}\left(d_{0}\right)$ is the received power from the link source located at distance $d_{0}, N_{0}$ is the power noise spectral density, $B$ the channel bandwidth, and $\mathbf{P}_{\text {int }}$ is the total interference power at the link receiver, i.e., the sum of the instantaneous received powers from all the undesired transmitters. More precisely, in an indoor environment, one has

$$
\mathbf{P}_{\text {int }}^{(\text {indoor })} \triangleq \sum_{i=1}^{N_{\text {close }}} \boldsymbol{\Lambda}_{i} \mathbf{P}_{i}\left(d_{i}\right)+\sum_{j=1}^{N_{\text {far }}} \boldsymbol{\Lambda}_{j} \mathbf{P}_{\text {env }}
$$

and, in an outdoor environment, one has

$$
\mathbf{P}_{\text {int }}^{\text {(outdoor) }} \triangleq \sum_{k=1}^{N_{\text {out }}} \boldsymbol{\Lambda}_{k} \mathbf{P}_{k}\left(d_{k}\right) .
$$

Finally, as typical in the context of BANs, we assume that all nodes use the same transmit power, i.e., $P_{i}(0)=$ $P_{j}(0)=P_{k}(0)=P_{0}(0), \forall i, j, k$. 


\section{A. Link probability of success with short-range transmission in indoor scenarios}

The link probability of success for a required threshold SINR value $\theta$ in the context of a short, indoor, log-normal faded link is equal to

$$
\begin{aligned}
\mathcal{P}_{\text {close }}^{(\text {indoor })} & =\mathbb{P}\{\text { SINR }>\theta\} \\
& =\mathbb{E}_{\mathbf{P}_{\text {int }}}\left[\mathbb{P}\left\{\frac{P_{0} L\left(d_{0}\right) \mathbf{X}_{0}}{N_{0} B+P_{\text {int }}}>\theta \mid \mathbf{P}_{\text {int }}^{(\text {indoor })}\right\}\right] \\
& =\mathbb{E}_{X, \Lambda, P_{\text {env }}}\left[1-\mathbb{P}\left\{\mathbf{X}_{0} \leq \theta \frac{N_{0} B+\mathbf{P}_{\text {int }}^{(\text {indoor })}}{P_{0} L\left(d_{0}\right)}\right\}\right] \\
& =\mathbb{E}_{X, \Lambda, P_{\text {env }}}\left[\frac{1}{2}+\frac{1}{2} \operatorname{erf}\left(\frac{-10}{\sigma \sqrt{2}} \log _{10}\left(\theta \frac{N_{0} B+\mathbf{P}_{\text {int }}^{(\text {indoor })}}{P_{0} L\left(d_{0}\right)}\right)\right)\right] .
\end{aligned}
$$

In the Appendix, it is shown that

$$
\zeta(z ; \sigma) \triangleq \frac{1}{2}+\frac{1}{2} \operatorname{erf}\left(\frac{-10 \log _{10} z}{\sigma \sqrt{2}}\right) \approx \sum_{m}^{n} c_{m} \exp \left(-a_{m} z\right),
$$

where $\left\{c_{m}\right\}_{m=1}^{n}$ and $\left\{a_{m}\right\}_{m=1}^{n}, n$ being an integer determined by the expansion accuracy, are suitable coefficients. By using the function $\zeta(\cdot ; \cdot)$ and recalling expression (10) for the interference power, the link probability of success (12) can be written as follows:

$$
\begin{aligned}
\mathcal{P}_{\text {close }}^{\text {(indoor) }}= & \mathbb{E}\left[\zeta\left(\theta \frac{\mathbf{P}_{\text {int }}^{(\text {indoor })}}{P_{0} L\left(d_{0}\right)} ; \sigma\right)\right] \\
= & \sum_{m=1}^{n} c_{m} \exp \left(\frac{-a_{m} \theta N_{0} B}{P_{0} L\left(d_{0}\right)}\right) \\
& \times \mathbb{E}\left[\exp \left(-a_{m} \theta \sum_{i=1}^{N_{\text {close }}} \frac{L\left(d_{i}\right)}{L\left(d_{0}\right)} \mathbf{X}_{i} \boldsymbol{\Lambda}_{i}\right)\right] \\
& \times \mathbb{E}\left[\exp \left(-a_{m} \theta \sum_{j=1}^{N_{\text {far }}} \frac{\mathbf{P}_{\text {env }}}{P_{0} L\left(d_{0}\right)} \mathbf{\Lambda}_{j}\right)\right],
\end{aligned}
$$

where, in the last passage, we have used the fact that the RVs $\left\{\boldsymbol{\Lambda}_{i}, \boldsymbol{\Lambda}_{j}, \mathbf{P}_{\text {env }}, X_{i}\right\}$ are independent. The term in the third line of the expression at the right-hand side of (13) can be further expressed as

$$
\begin{aligned}
\mathbb{E} & {\left[\exp \left(-a_{m} \theta \sum_{i=1}^{N_{\text {close }}} \frac{L\left(d_{i}\right)}{L\left(d_{0}\right)} \mathbf{X}_{i} \boldsymbol{\Lambda}_{i}\right)\right] } \\
= & \prod_{i=1}^{N_{\text {close }}} \mathbb{E}\left[\exp \left(-a_{m} \theta \frac{L\left(d_{i}\right)}{L\left(d_{0}\right)} \mathbf{X}_{i} \boldsymbol{\Lambda}_{i}\right)\right] \\
= & \prod_{i=1}^{N_{\text {close }}}\left\{\mathbb{P}\left\{\boldsymbol{\Lambda}_{i}=0\right\} \times 1+\mathbb{P}\left\{\boldsymbol{\Lambda}_{i}=1\right\}\right. \\
& \left.\times \mathbb{E}\left[\exp \left(-a_{m} \theta \frac{L\left(d_{i}\right)}{L\left(d_{0}\right)} \mathbf{X}_{i}\right)\right]\right\} \\
= & \prod_{i=1}^{N_{\text {close }}} q \int_{0}^{\infty} \exp \left(-a_{m} \theta 10^{\gamma\left(d_{i}-d_{0}\right)} x\right) f_{\mathbf{X}}(x) \mathrm{d} x+(1-q) .
\end{aligned}
$$

The final integral expression in (15) can be numerically computed. The term in the third line of expression (13) can be expressed as follows:

$$
\begin{aligned}
& \mathbb{E}\left[\exp \left(-a_{m} \theta \sum_{j=1}^{N_{\text {far }}} \frac{\mathbf{P}_{\text {env }}}{P_{0} L\left(d_{0}\right)} \boldsymbol{\Lambda}_{j}\right)\right] \\
& =\prod_{j=1}^{N_{\text {far }}} \mathbb{E}\left[\exp \left(-a_{m} \theta \frac{\mathbf{P}_{\text {env }}}{P_{0} L\left(d_{0}\right)} \mathbf{\Lambda}_{j}\right)\right] \\
& =\prod_{j=1}^{N_{\text {far }}}\left\{\mathbb{P}\left\{\boldsymbol{\Lambda}_{j}=0\right\} \times 1+\mathbb{P}\left\{\boldsymbol{\Lambda}_{j}=1\right\}\right. \\
& \left.\times \mathbb{E}\left[\exp \left(-a_{m} \theta \frac{\mathbf{P}_{\text {env }}}{P_{0} L\left(d_{0}\right)}\right)\right]\right\} \\
& =\left[(1-q)+q \int_{0}^{\infty} \exp \left(-a_{m} \theta \frac{x}{P_{0} L\left(d_{0}\right)}\right) \frac{1}{P_{\text {env }}} e^{-x / P_{\text {env }}} \mathrm{d} x\right]^{N_{\text {far }}} \\
& =\left[1-\frac{\theta q}{\frac{L_{0} 10^{\gamma d_{0}}}{L^{(\text {env })}}+\theta}\right]^{N_{\text {far }}} \text {. }
\end{aligned}
$$

Finally, by inserting (15) and (16) into (13), the link probability of success can be given by the expression in (17).

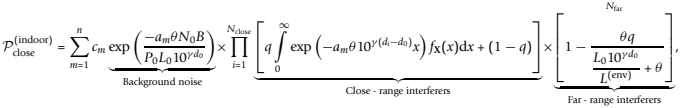

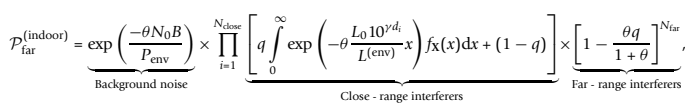

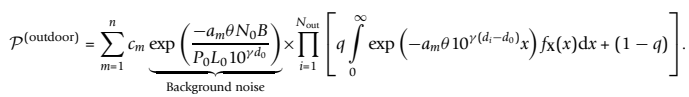

\section{B. Link probability of success with long-range} transmission in indoor scenarios

The Rayleigh-faded channel model applies to indoor links with length $d>d_{\text {cross }}$. In this scenario, $\mathbb{E}[\mathbf{P}(d)] \approx P_{\text {env }}$ (for both the intended transmitter and interferers) and the link probability of success can be expressed as follows:

$$
\begin{aligned}
\mathcal{P}_{\text {far }}^{(\text {indoor })=} & \mathbb{P}\{\text { SINR }>\theta\} \\
= & \mathbb{E}_{\mathbf{P}_{\mathrm{mt}}}\left[\mathbb{P}\{\text { SINR }>\theta\} \mid \mathbf{P}_{\text {int }}^{(\text {indoor })}\right] \\
= & \mathbb{E}\left[\exp \left\{-\frac{\theta\left(N_{0} B+\mathbf{P}_{\text {int }}^{(\text {indoor })}\right)}{P_{\text {env }}}\right\}\right] \\
= & \exp \left(\frac{-\theta N_{0} B}{P_{\text {env }}}\right) \\
& \times \mathbb{E}\left[\exp \left(-\theta \sum_{i=1}^{N_{\text {close }}} \frac{P_{i} L\left(d_{i}\right)}{P_{\text {env }}} \mathbf{X}_{i} \boldsymbol{\Lambda}_{i}\right)\right] \\
& \times \mathbb{E}\left[\exp \left(-\theta \sum_{j=1}^{N_{\text {far }}} \frac{\mathbf{P}_{\text {env }}}{P_{\text {env }}} \boldsymbol{\Lambda}_{i}\right)\right] .
\end{aligned}
$$


It can be observed that the terms in the second and third lines at the right-hand side of (20) are similar to (15) and (16). Therefore, by using the same derivation of Section 3-A, with $P_{0} L\left(d_{0}\right)$ replaced by $P_{0} L^{(\text {env })}$, one has

$$
\begin{aligned}
& \mathbb{E}\left[\exp \left(-\theta \sum_{i=1}^{N_{\text {close }}} \frac{P_{i} L\left(d_{i}\right)}{P_{\text {env }}} \mathbf{X}_{i} \boldsymbol{\Lambda}_{i}\right)\right] \\
& =\prod_{i=1}^{N_{\text {close }}}\left[q \int_{0}^{\infty} \exp \left(-\theta \frac{L_{0} 10^{\gamma d_{i}}}{L^{(\text {env })}} x\right) f_{\mathbf{X}}(x) \mathrm{d} x+(1-q)\right]
\end{aligned}
$$

and

$$
\mathbb{E}\left[\exp \left(-\theta \sum_{j=1}^{N_{\text {far }}} \frac{\mathbf{P}_{\text {env }}}{P_{\text {env }}} \boldsymbol{\Lambda}_{i}\right)\right]=\left[1-\frac{\theta q}{1+\theta}\right]^{N_{\text {far }}} .
$$

By inserting (21) and (22) into (20), one obtains the final expression (18) for the probability of successful transmission on the link.

\section{Link probability of success in outdoor scenarios}

In these scenarios, the links are subject to log-normal fading, and exponential power decreases. The link probability of success can simply be derived using the derivation in Section 3-A, setting $N_{\text {out }}=N_{\text {close }}$ and $N_{\text {far }}=0$ (this does not mean that there are not far interferers, but that their propagation model is simply the same of close interferers). Therefore, the computation of the link probability of success $\mathcal{P}$ (outdoor) is straightforward from (17) and the final expression is given in (19).

\section{Minimum transmit powers}

The first terms in the sum at the right-hand side of (17) and the first multiplicative term at the right-hand side of (18) correspond to the link probabilities of success in a noise-limited regime, i.e., when no interferers are present. In fact, setting $N_{\text {close }}=N_{\text {far }}=0$ (i.e., $\mathbf{P}_{\text {int }}=0$ ) in (17) and (18), the probabilities of successful link transmission reduce to

$$
\begin{array}{ll}
\mathcal{P}_{\text {close }}^{\text {(indoor) }}=\zeta\left(\frac{\theta N_{0} B}{P_{0} L_{0} 10^{\gamma d_{0}}}\right) & \text { if } d<d_{\text {cross }} \\
\mathcal{P}_{\text {far }}^{\text {(indoor) }}=\exp \left(-\frac{\theta N_{0} B}{P_{\text {env }}}\right) \quad \text { if } d \geq d_{\text {cross }} .
\end{array}
$$

Therefore, if a threshold link probability of success equal to $\mathcal{P}_{\text {th }} \in(0,1)$ is required, the minimum required transmit power in an indoor scenario can be written as follows ${ }^{\mathrm{c}}$ :

$$
P_{0}^{\text {(indoor) }} \geq \begin{cases}\frac{\theta k_{\mathrm{b}} T B}{L_{0} 10^{\gamma d_{0}} \zeta^{-1}\left(\mathcal{P}_{\text {th }}\right)} & \text { if } d<d_{\text {cross }} \\ -\frac{\theta k_{\mathrm{b}} T B}{\ln \mathcal{P}_{\text {th }}} & \text { if } d \geq d_{\text {cross }}\end{cases}
$$

where $N_{0}$ has been expressed as $T k_{\mathrm{b}}$, with $T$ being the room temperature (dimension: $[\mathrm{K}]$ ) and $k_{\mathrm{b}}=1.38 \times 10^{-}$ ${ }^{23} \mathrm{~J} / \mathrm{K}$ being the Boltzman's constant, and $B$ being the transmission bandwidth.

In an outdoor scenario, by setting $N_{\text {out }}=0$ in (19), the probability of successful link transmission reduces to

$$
\mathcal{P}^{\text {(outdoor) }}=\zeta\left(\frac{\theta N_{0} B}{P_{0} L_{0} 10^{\gamma d_{0}}}\right) .
$$

Considering a threshold link probability of success equal to $\mathcal{P}_{\text {th }}$, the minimum required transmit power becomes

$$
P_{0}^{\text {(outdoor) }} \geq \frac{\theta k_{\mathrm{b}} T B}{L_{0} 10^{\gamma d_{0}} \zeta^{-1}\left(\mathcal{P}_{\mathrm{th}}\right)}
$$

In Figure 6, the minimum required transmit power $P_{0}$ for a successful link transmission in an indoor scenario with a ZigBee equipment $(B=5 \mathrm{MHz}, \theta=5 \mathrm{~dB})$, operating at $T=300 \mathrm{~K}$ and with log-normal fading characterized by $\sigma_{\mathrm{dB}}=8 \mathrm{~dB}$, is shown as a function of the distance, considering various values of the required link probability of success of $\mathcal{P}_{\text {th }}$. As expected, once the link distance overcomes the critical value around $25 \mathrm{~cm}$, the required transmit power becomes constant. The dashed region corresponds to the typical operational region. In Figure 7, the minimum required transmit power for an outdoor scenario is shown as a function of the distance. The system parameters are set as in Figure 6. It can be observed that, unlinke an indoor scenario, in an outdoor scenario, the minimum required transmit power is an increasing function of the distance (in fact, there are no reflections from surrounding objects).

On the basis of the results presented in Figures 6 and 7 , the following observations can be made. The value of $\mathcal{P}_{\text {th }}$ plays a limited role on the minimum transmit

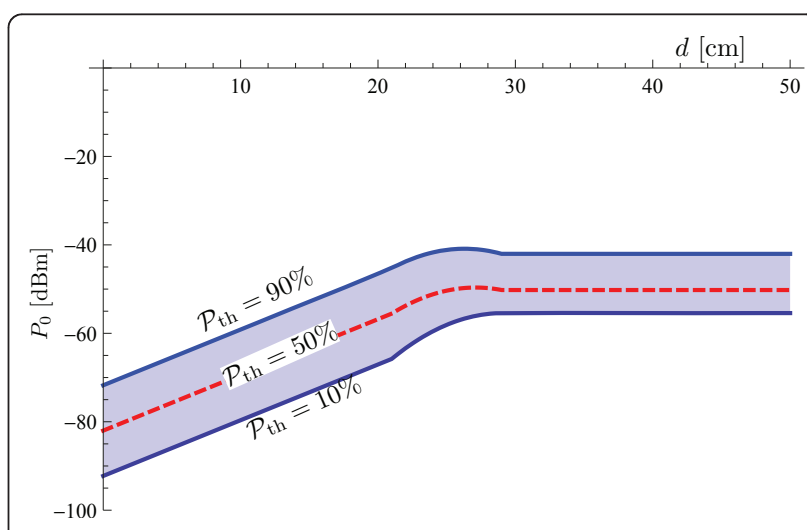

Figure 6 Minimum transmit power as a function of the distance in an indoor scenario. The dashed region is the operational region of a BAN. 


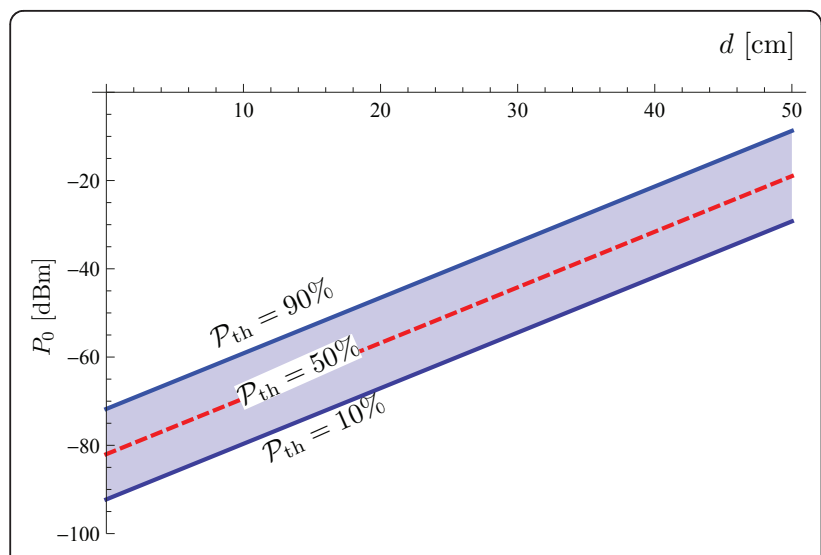

Figure 7 Minimum transmit power as a function of the distance in an outdoor scenario. The dashed region is the operational region of a BAN.

power. If the transmit power is constrained by energy concerns, then only short-range communications (some tenths of centimeters) will be possible: therefore, a multi-hop network architecture is to be preferred. Finally, in an indoor environment, as seen from Figure 6 , the reflections from the surrounding environment make the minimum transmit power become constant when $d \geq 25 \mathrm{~cm}$.

In the remainder of this study, we will consider only interference-limited BANs, i.e., scenarios where condition (23) is satisfied. Formally, this is equivalent to assuming that $N_{0} B \ll \mathbf{P}_{\text {int }}$.

\section{Tree topologies and multi-hop communications}

\section{A. BAN tree topologies}

In [35], a preliminary performance analysis of BANs with star topologies was carried out. Indeed, these topologies are well suited for medical applications since they exhibit low-power consumption [36] and can perform application-specific data aggregation [37-39]. However, in order to limit the transmit power, the use of tree (hierarchical) BAN topologies is appealing.
In Figure 8, an illustrative tree topology is presented. It can be observed that, in a generic situation, multiple hierarchical levels have to be considered because of the existence of multiple measurement clusters. Each cluster has a cluster-head, which collects the data from its sensors (and its own data) and transmits them to the final sink. We assume that the links in each cluster are short (i.e., each cluster is in a regime of close-range interferers) and the links from the cluster-head to the coordinator are long (i.e., there is a regime of far-range interferers). However, the proposed framework is applicable to any type of tree architecture.

In this article, we will focus on the impact of the tree clustering on the throughput and energy consumption. More precisely, in Figure 9, three two-level (i.e., 3-tier) hierarchical topologies with 16 nodes are presented.

\section{B. Medical applications of the tree topologies}

The three topologies shown in Figure 9 are generic and suitable for a range of medical applications $[40,41]$. More precisely, "Configuration A" refers to a multi-sensor site where highly dense clusters of nodes are deployed. This is representative of medical scenarios where intense monitoring, in a few areas of interest, is needed. Relevant medical applications are mobile EEG (ElectroEncephaloGraphy) or post-operative monitoring of localized critical health conditions.

The second configuration ("Configuration B") is more balanced and corresponds to multiple monitoring sites distributed over the body. Two typical BAN scenarios are encompassed: (i) redundant acquisitions of local physiological signals (for safety reasons) and (ii) multiple independent sensing devices, each having its own relay node (i.e., ECG (ElectroCardioGraphy) combined with limbs monitoring and motion sensors). Relevant medical applications include stroke or Parkinson's disease monitoring (through a combination of EEG, accelerometers, and a gyroscope), and cardiac arrest or ischaemic heart disease monitoring (through a combination of an ECG and a mechanoreceptor).

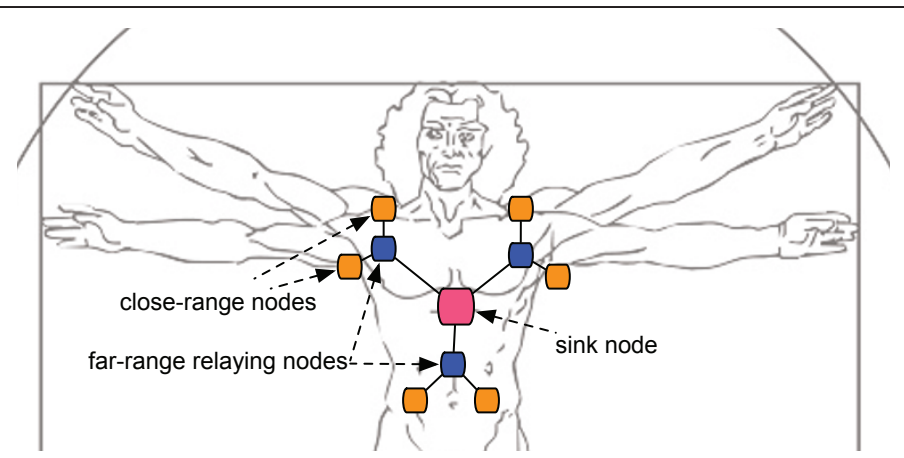

Figure 8 Central sink (in red) surrounded by far-range relaying nodes (in blue). These relays connect close-range medical sensor nodes (in orange). 


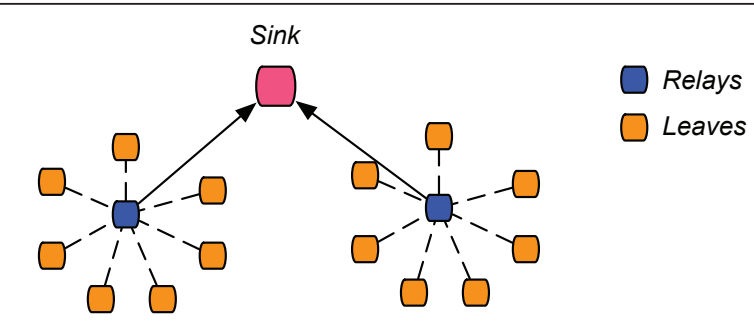

(a) Configuration A: 7 leaves at each of the 2 relays $\left(N_{1}=7, N_{2}=2\right)$

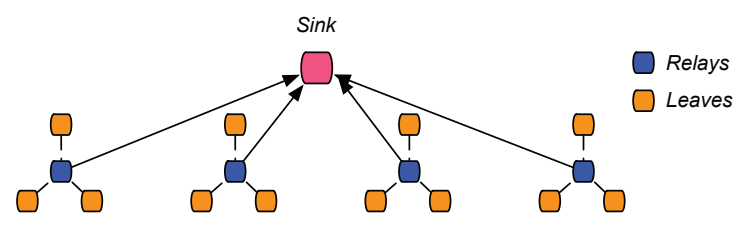

(b) Configuration B: 3 leaves at each of the 4 relays $\left(N_{1}=3, N_{2}=4\right)$

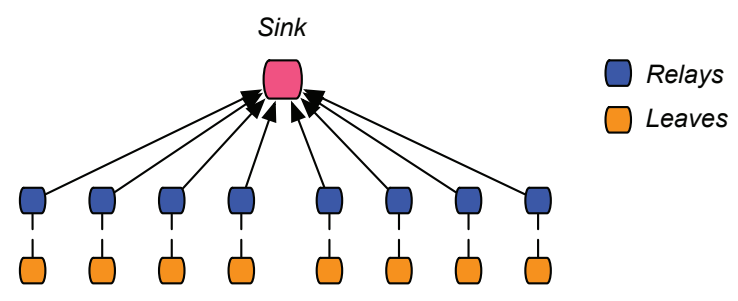

(c) Configuration C: 1 leaf at each of the 8 relays $\left(N_{1}=1, N_{2}=8\right)$

Figure 9 3-tier hierarchical BANs with 16 sensor nodes (leaves): three possible configurations are considered. (a) Configuration A: seven leaves at each of the two relays $\left(N_{1}=7, N_{2}=2\right) ;(\mathbf{b})$ Configuration B: three leaves at each of the four relays $\left(N_{1}=3, N_{2}=\right.$ 4); and (c) Configuration C: one leaf at each of the eight relays $\left(N_{1}\right.$ $\left.=1, N_{2}=8\right)$.

The third configuration ("Configuration C") is representative of a generic sensing scheme where multiple sensors are networked and distributed all over the body without local clustering. In this sense, it is representative of a star topology, as each intermediate relay is connected to a single sensing unit. A relevant medical application is given by a wearable vest with multiple sensors across it (each node may measure local blood pressure, collect electrical signals for ECG, and measure local accelerations).

\section{Multi-hop traffic model}

In this study, we consider a slotted communication model, where $T_{\text {slot }}$ (dimension: [s]) denotes the duration of each slot. It is important to distinguish between data generation and data transmissions at the sensors. Data generation, in real applications, depends on the quantity to be measured; data transmission depends on the communication system design. We now show clearly that generation and transmission cross-influence each other.
Let us first model data generation. For the sake of simplicity, we assume that, in each slot, a sensor can generate at most one packet, and we denote by $\lambda \in[0$, 1] the corresponding probability of packet generation. In other words, the number of packets generated by a sensor in a slot is a Bernoulli RV with parameter $\lambda$, i.e., $\lambda$ can also be interpreted as the average number of packets generated in a slot. Therefore, $\lambda / T_{\text {slot }}$ represents the average number of generated packets per unit time (dimension: $\left[\mathrm{s}^{-1}\right]$ ). Finally, denoting the packet length as $L$ (dimension: [b/pck]) and the (fixed) transmission data-rate as $R_{\mathrm{b}}$ (dimension: [b/s]), the packet duration is $T_{\mathrm{pck}} \triangleq L / R \mathrm{~b}$ (dimension [s]). For stability reasons, it has to hold that

$$
T_{\text {pck }} \leq T_{\text {slot }} .
$$

Given specific transmission technology (which determines $R_{\mathrm{b}}$ ) and communication protocol, e.g., Zigbee (which determines the percentage of overhead in a transmitted packet), it is possible to determine the maximum payload per slot by imposing $T_{\mathrm{pck}}=T_{\text {slot }}$. Denoting $L_{\text {payload }}<L$ the length of the payload and by $r_{\text {samp- }}$ med the sampling rate of the medical sensor (dimension: $[\mathrm{b} / \mathrm{s}])$, the following condition has to hold:

$$
r_{\text {samp-med }} \leq \frac{L_{\text {pay1oad }}}{T_{\text {s1ot }}}
$$

In other words, the above inequality shows clearly that the communication/networking technology has an impact on the features of the (medical) sensors. We remark that a careful analysis of the transmission probabilities of the (medical) sensors will more likely lead to different values of $\lambda$ (and $q$ ) for each node, depending on the type of physiological constant and the congestion at the relays, among others. This analysis goes beyond the scope of this article and is the subject of future research. However, whatever the used sensors, it is possible to derive the equivalent value of $\lambda$ and, therefore, rely on the proposed framework.

At this point, we model data transmission. Under the considered assumption of slotted ALOHA MAC protocol, a simplified model for the MAC protocol, a sensor has probability $q$ of transmitting a packet in a slot. Obviously, this makes sense only if the node has a packet to transmit. Moreover, for stability reasons, it has to hold that

$$
\lambda \leq q .
$$

In fact, the condition $\lambda>q$ would be equivalent to assuming that the sensor generates, per time unit, more packets than those it can actually transmit. In this case, there would be an overflow at the sensor, and packets would be lost. On the other hand, assuming $\lambda<q$ is 
meaningless as well, it is impossible that the transmission probability of a sensor node is higher than its generation probability (what would it transmit?). Therefore, in the considered simplified model, it follows that $\lambda=q$, i.e., the generation and transmission processes coincide. Note also that, according to this model, $q$ is equal to the per-node load (defined as the average number of packets generated during an interval equal to the duration of a packet transmission). Therefore, the network load $G$ (adimensional) is simply equal to $q \cdot N_{\text {tot }}$, where $N_{\text {tot }}$ denotes the total number of sensor nodes in the BAN.

Let $\mathcal{N}$ be the set that consists of $N$ leaf sensor nodes connected to a given relay node (i.e., the set of sensor nodes per cluster, excluding the relay). In half-duplex communications, a node transmits if and only if (i) it has data to send or (ii) it has no data to send but acts as a relay for other nodes. We denote by $q_{\text {leaf }}$ the probability that a leaf node has data to send. Obviously, $q_{\text {leaf }}$ $=q$, i.e., the probability that data are present and ready to be sent. A relay node will transmit if it gets data from a leaf (event denoted as "relay") or has to send sensed information (event denoted as "data"), i.e.,

$$
\begin{aligned}
q_{\text {relay }} & =\mathbb{P}\{\text { data } \vee \text { relay }\} \\
& =\mathbb{P}\{\text { data }\}+\mathbb{P}\{\text { relay }\}-\mathbb{P}\{\text { data }\} \mathbb{P}\{\text { relay }\}
\end{aligned}
$$

where, in the last passage, we have exploited the fact that the events "data" and "relay" are independent. By definition, $\mathbb{P}\{$ data $\}=q$. Obviously, the probability of relaying depends on (i) the probability of having data present at any node and (ii) their successful reception at the relaying node. Therefore,

$$
\begin{aligned}
\mathbb{P}\{\text { relay }\} & =\mathbb{P}\{\exists n \in \mathcal{N}: \operatorname{transmit}(n) \wedge \operatorname{successful}(n)\} \\
& =1-\mathbb{P}\{\forall n \in \mathcal{N}, \neg(\operatorname{transmit}(n) \wedge \operatorname{successful}(n))\} \\
& =1-\prod_{i=1}^{N}\left(1-q_{1 \text { eaf }} \mathcal{P}_{\text {leaf } \rightarrow \text { relay }}^{(i)}\right) .
\end{aligned}
$$

According to the assumption at the end of Section 3, a transmission is successful if the channel is not in an outage, i.e., if the (instantaneous) SINR exceeds a certain threshold $\theta$. Therefore, $\mathcal{P}_{\text {1eaf } \rightarrow \text { relay }}=\mathbb{P}\{$ SINR $>\theta\}$ on the considered link. Since (i) all links in a cluster are, on average, equal and (ii) $q_{\text {leaf }}=q$, one has

$$
\mathbb{P}\{\text { relay }\}=1-\left(1-q \mathcal{P}_{1 \text { eaf } \rightarrow \text { relay }}\right)^{N} .
$$

Finally, $\mathbb{P}\{$ data $\}=q$, from (25), one has

$$
q_{\text {relay }}=q+(1-q)\left[1-\left(1-q \mathcal{P}_{\text {leaf } \rightarrow \text { relay }}\right)^{N}\right],
$$

where $\mathcal{P}_{1 \text { eaf } \rightarrow \text { relay }}$ can be either (17) or (19), in indoor or outdoor scenarios, respectively.

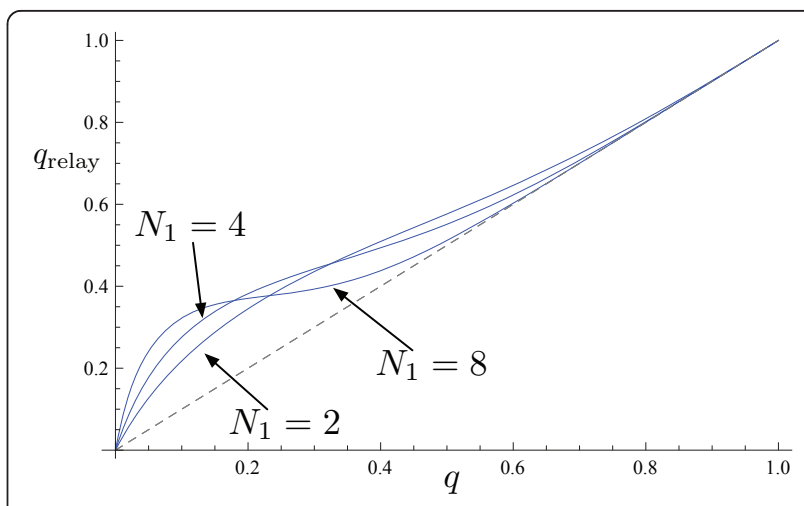

Figure 10 Terminal probability of transmission of a relay node as a function of a single terminal probability of transmission and for $N L\{2,4,8\}$ neighbor nodes and $\mathcal{P}_{\text {leaf } \rightarrow \text { relay }}=1$.

In Figure 10, the probability of transmission of a relay node is shown as a function of the probability of transmission of a single node, considering various values for the number $N_{1}$ of nodes in a first-level cluster (i.e., leaves of the collection tree). It can be observed that when $q \leq$ 0.5 , the value $q_{\text {relay }}$ depends on the relaying (i.e., $q_{\text {relay }} \geq q$ since it accounts for the traffic of the leaves plus the traffic generated by the relay) and, when $q>0.5$, it is dominated by the relay probability of sending the data itself (i. e., $q_{\text {relay }} \approx q$ since the relay transmits its data and prohibits reception of the ones from the leaves).

Finally, in multiple-tier topologies (more complex than the 3-tier considered in this article), the same approach can be applied to compute the probability of transmission of any node acting as a relay at a given hierarchical level of the network. In the considered 3-tier topologies, this approach can be straightforwardly applied to evaluate $q_{\text {sink }}$, i.e., the probability of transmission from the sink (e.g., through a $3 G$ connection).

\section{Network-level performance analysis}

The main simulation parameters are set as follows. With reference to the topologies in Figure 9, the distances between a leaf and its relay and between a relay and the sink are 10 and $30 \mathrm{~cm}$, respectively. The SINR threshold is set to $\theta=5 \mathrm{~dB}$. The fading power of the lognormal propagation model is $\sigma_{\mathrm{dB}}=8 \mathrm{~dB}$. These values correspond to typical, multi-kpbs sensor nodes.

\section{A. Performance metrics}

In the following, we will consider two key performance metrics: (i) the link-level throughput, and (ii) the energy consumption rate.

Regarding the link-level throughput, a transmission will be successful if and only if a transmission link is not 
in an outage. This corresponds to requiring that the (instantaneous) SINR of the link is above the threshold $\theta$, i.e.,

$$
\mathcal{P}=\mathbb{P}\{\text { SINR }>\theta\}
$$

where $\mathcal{P}$ is either equal to $\mathcal{P}_{\text {far }}^{\text {(indoor) }}, \mathcal{P}_{\text {close }}^{\text {(indoor) }}$, or $\mathcal{P}$ (outdoor) depending on the scenario at hand. The probabilistic link throughput [42] (adimensional) of a node is defined as follows:

- in the full-duplex communication case, it corresponds to the product of (i) $\mathcal{P}$ and (ii) the probability that the transmitter actually transmits (i.e., q);

- in the half-duplex communication case, it corresponds to the product of (i) $\mathcal{P}$, (ii) the probability that the transmitter actually transmits (i.e., $q$ ), and (iii) the probability that the receiver actually receives (i.e., 1 - q).

The probabilistic link throughput can be interpreted as the unconditioned reception probability which can be achieved with a simple automatic-repeat-request scheme with error-free feedback [43]. For the slotted ALOHA transmission scheme under consideration in the context of BAN (where transmissions can typically be organized in a full-duplex way), the probabilistic throughput is

$$
\tau \triangleq q \times \mathcal{P} \text {. }
$$

More specifically, in our analyses, three different nodes are considered: leaves, relays, and the sink. The corresponding throughput metrics are

$$
\begin{aligned}
& \tau_{\text {leaf }}=q_{\text {leaf }} \times \mathcal{P}_{\text {1eaf } \rightarrow \text { relay }}=q \times \mathcal{P}_{\text {1eaf } \rightarrow \text { relay }}, \\
& \tau_{\text {relay }}=q_{\text {relay }} \times \mathcal{P}_{\text {relay } \rightarrow \text { sink }} . \\
& \tau_{\text {sink }}=q_{\text {sink }} \times 1=q_{\text {sink }},
\end{aligned}
$$

since it is supposed that the sink acts as a special device and can communicate with the external equipments with probability equal to one (e.g., it is used to store data on a memory card or to send these data by means of a reliable transmission technology, such as, for example, 3G).

The second performance metric of interest is the energy consumption rate. The average energy consumed by the network in a slot, denoted as $E$ (dimension: [J]), can be expressed as

$$
E=q N_{\text {leaves }} E_{\mathrm{TX}}+q_{\text {relay }} N_{\text {relays }}\left(E_{\mathrm{TX}}+E_{\mathrm{RX}}\right)+q_{\text {sink }} E_{\mathrm{RX}}
$$

where $E_{\mathrm{TX}}$ and $E_{\mathrm{RX}}$ are the energies (dimension: [J]) consumed by single-packet transmission and reception acts, respectively.
In most wireless systems, $E_{\mathrm{TX}} \approx E_{\mathrm{RX}}$, and one has

$$
\begin{aligned}
E & =E_{\mathrm{TX}} \times\left(q N_{\text {leaves }}+2 q_{\text {relay }} N_{\text {relays }}+q_{\text {sink }}\right) \\
& =E_{\mathrm{TX}} \times \mathcal{E},
\end{aligned}
$$

where $\mathcal{E} \triangleq E / E_{\mathrm{TX}}$ is denoted as energy depletion rate (adimensional) and corresponds to the ratio of the energy consumed by the network in a slot and the energy consumed to transmit a single packet.

We now provide the reader with a performance analysis of all the three hierarchical topologies deployed in outdoor and indoor scenarios.

\section{B. Outdoor Scenarios}

In Figure 11, the per-node throughputs at the three hierarchical levels (i.e., leaf nodes, relaying nodes, and central sink) are shown as functions of the sensors' probability of transmission $q$. The three subfigures refer to the three topologies in Figure 9. It can be observed that the three considered topologies lead to very different performances (in terms of throughput) for the leaves, the relays, and the sink.

In the Configuration A (Figure 9a) the throughputs of the leaves, relays, and sink are increasing functions of $q$ for small values of $q$ and reach the maximum values at $q \simeq 0.15$. For $q>0.15, \tau_{\text {leaf }}$ starts decreasing. In contrast, $\tau_{\text {relay }}$ remains approximately constant for $q \in(0.15$, 0.45 ): in fact, the packets' losses at the leaves' are compensated by data transmitted by the relay node itself, so that the overall value of $\tau_{\text {relay }}$ tends to remain stable. It can also be observed that the throughput at the sink remains approximately constant for $q \in(0.15,0.45)$, and its value is close to the maximum achievable throughput of any slotted ALOHA system without lossy links, which is $e^{-1} \approx 0.37$ [44]. Since the throughput at the sink can be interpreted as the overall network throughput, it can be concluded that the network Configuration A yields an excellent channel utilization at the sink node. Regarding the operational region of this configuration, it can be seen from Figure 11a that for $q \geq 0.6$ one has $\tau_{\text {leaf }} \simeq 0$, i.e., the load is too high, and the leaves tend to be disconnected from the network, i.e., packets from the leaves are no longer relayed and successfully transmitted to the sink. Consequently, for $q \geq 0.6$, the throughputs at the relays and at the sink tend to decrease.

The performance with the second topology-referred to as Configuration B (Figure 9b)-is analyzed in Figure 11b. It can be observed that the throughput at the relays is, for small values of $q$, an increasing function of $q$ and reaches a maximum value at $q \approx 0.1$. Beyond this value, the throughput at the relays tend to rapidly decrease. On the other hand, $\tau_{\text {leaf }}$ is an increasing function till $q \approx$ 0.3 : this is because the number of sensors per cluster (3) is smaller than the number of relays (4) and, therefore, 


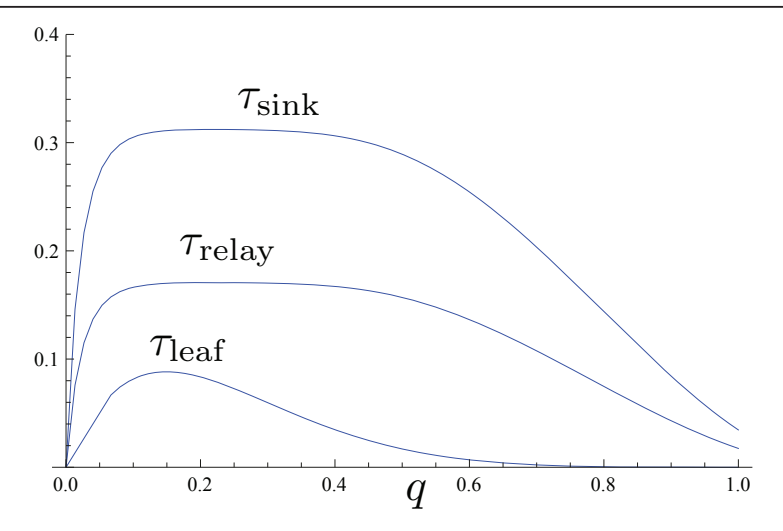

(a) Configuration A: 7 leaves at each of the 2 relays $\left(N_{1}=7, N_{2}=2\right)$.

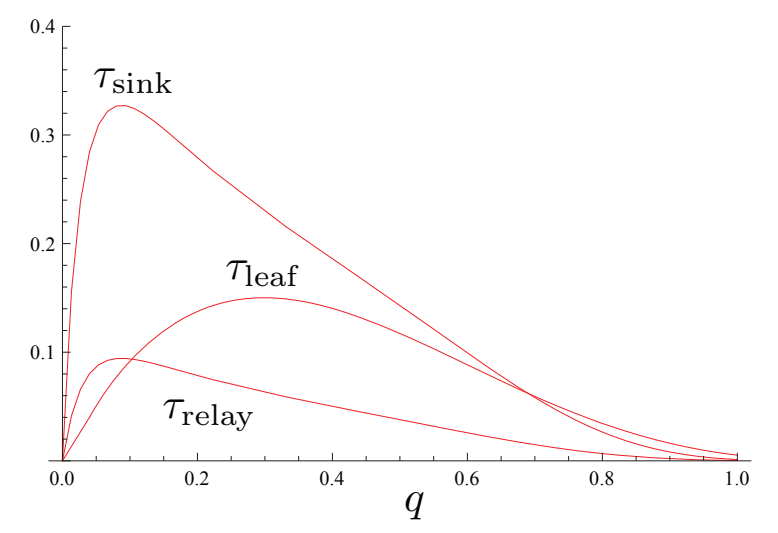

(b) Configuration B: 3 leaves at each of the 4 relays $\left(N_{1}=3, N_{2}=4\right)$.

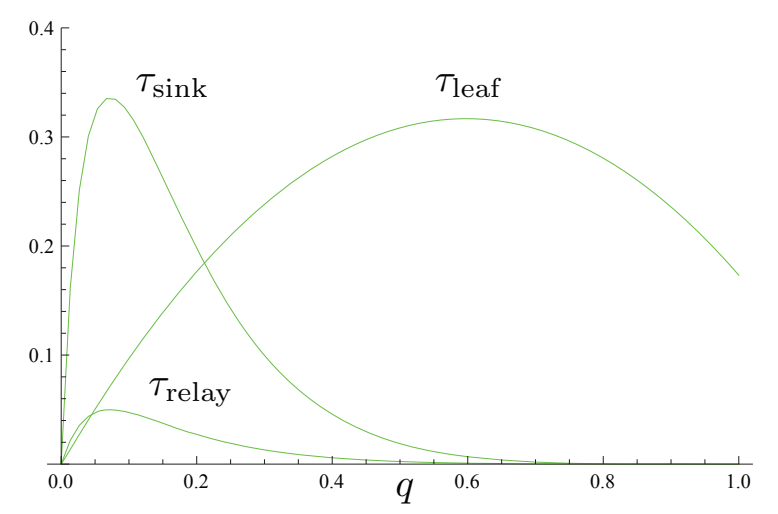

(c) Configuration C: 1 leaf at each of the 8 relays $\left(N_{1}=1, N_{2}=8\right)$.

Figure 11 Per link throughputs for the three considered strategies in an outdoor environment. (a) Configuration $A$ : seven leaves at each of the two relays $\left(N_{1}=7, N_{2}=2\right)$; (b) Configuration $B$ : three leaves at each of the four relays $\left(N_{1}=3, N_{2}=4\right)$; and (c) Configuration C: one leaf at each of the eight relays $\left(N_{1}=1, N_{2}=8\right)$.

the throughput at the leaves continues to increase even if the throughput at the relays starts decreasing. Unlike Configuration $\mathrm{A}$, in Configuration $\mathrm{B}$, the maximum throughput at the leaves is higher than the maximum throughput at the relays. As the number of relays is relatively large, they tend to interfere with each other and, therefore, the throughput of the sink reaches a maximum at $q \approx 0.1$ and, then, decreases. It can be observed that the maximum throughput at the sink with Configuration B is approximately equal to that of Configuration A. However, unlike Configuration A, in Configuration $\mathrm{B}$, there is no interval of $q$ where the throughput at the sink tends to remain constant. In other words, this configuration does not support, at network level, a larger interval of values of $q$.

The last scheme-denoted as Configuration C (Figure 9c)-is highly centralized. Its performance is investigated in Figure 11c. As each cluster contains only one leaf node, $\tau_{\text {leaf }}$ is the highest. On the other hand, the relays interfere with each other while communicating to the sink and, therefore, $\tau_{\text {relay }}$ remains very low (its maximum value is around 0.05$)$. As a consequence, $\tau_{\text {sink }}$, after reaching its maximum value for $q \approx 0.08$ (similarly the previous configuration), tends to decrease to zero much faster than in Configuration B. Note that the maximum value of the throughput at the leaves is close to the maximum value of the throughput at the sink. Finally, note that for $q \geq 0.5$, even if $\tau_{\text {leaf }}$ is high, $\tau_{\text {sink }}$ is basically zero: in other words, no data transmitted by the leaves can be successfully transmitted by the sink to an external controller (e.g., through $3 G$ communications).

\section{Throughput in indoor scenarios}

In Figure 12, the per-node throughputs at the various hierarchical levels are presented for the three topologies of interest. As a first, general, observation, it is seen that the per-node throughputs are much lower in indoor scenarios than the corresponding ones, as shown in Figure 11 , in outdoor scenarios. This can be explained by the presence of a reflections off the limbs and the surrounding objects. Indeed, the initial antenna gain (at $d=d_{\text {ref }}$ ) is about $L_{\text {ref }}=-57.42 \mathrm{~dB}$, and this value is not very different from the gain of the environment, i.e., $L_{\mathrm{dB}}^{(\mathrm{env})}=-78 \mathrm{~dB}$. Therefore, short links are less affected (since the received signal power is much stronger than the reflected power), while longer links are more likely to suffer significant interference from the reflected waves. This was not the case in outdoor scenarios where distant nodes did not contribute to the interference thanks to the high path loss coefficient (i.e., $\gamma=$ $124 \mathrm{~dB} / \mathrm{m})$.

Regarding Configuration A, it is seen from Figure 12a that the leaves can support a wide range of values of $q$ (i.e., the throughput is non-zero for any value $q \in(0$, 0.6)). As anticipated in the description of the results in outdoor scenarios, the relays effectively accumulate the leaves' and their own data, guaranteeing the highest 


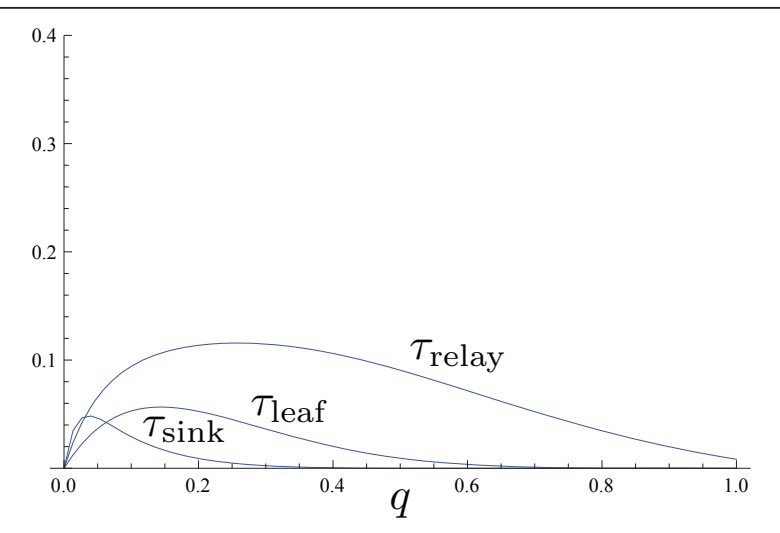

(a) Configuration A: 7 leaves at each of the 2 relays $\left(N_{1}=7, N_{2}=2\right)$.

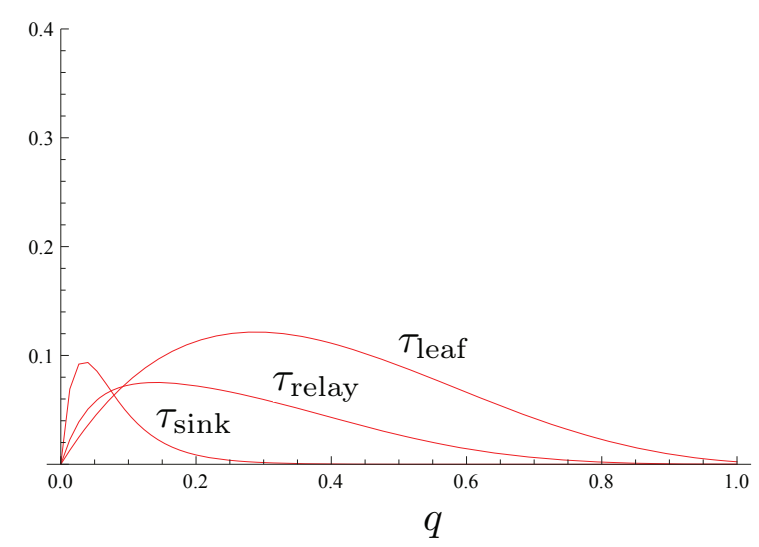

(b) Configuration B: 3 leaves at each of the 4 relays $\left(N_{1}=3, N_{2}=4\right)$.

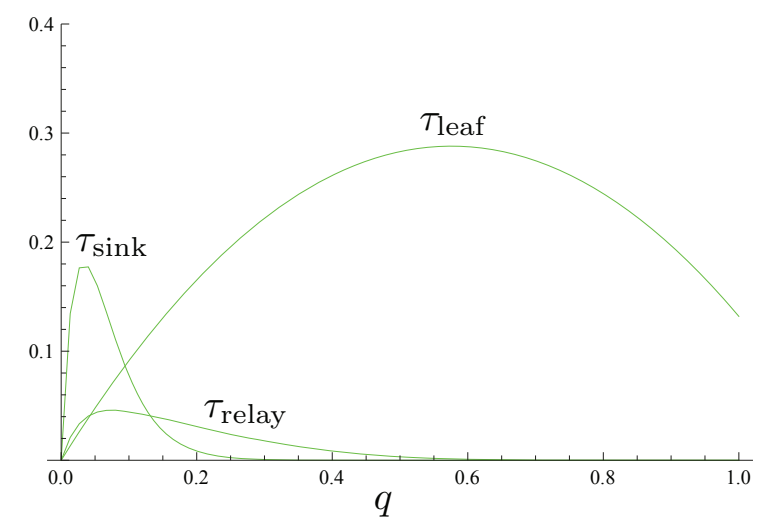

(c) Configuration C: 1 leaf at each of the 8 relays $\left(N_{1}=1, N_{2}=8\right)$.

Figure 12 Per-node throughputs (at each level) for the three considered topologies in an indoor environment. (a)

Configuration A: seven leaves at each of the two relays $\left(N_{1}=7, N_{2}\right.$

= 2); (b) Configuration B: three leaves at each of the four relays $\left(N_{1}\right.$

$\left.=3, N_{2}=4\right)$; and (c) Configuration C: one leaf at each of the eight relays $\left(N_{1}=1, N_{2}=8\right)$. throughput almost for all values of $q$-for very low values of $q, \tau_{\text {relay }}<\tau_{\text {sink }}$. However, the last links (i.e., the relayto-sink links) are subject to strong interference because of the reflections off the surrounding environment, and the sink throughput is much lower than in the outdoor scenario. More precisely, the throughput reaches a maximum at $q \approx 0.05$ and becomes insignificant for $q \geq 0.3$.

The performance of Configuration B is presented in Figure 12b. Since the tree is more balanced than in Configuration A (i.e., there are less leaves and more relays), the performance observed at the leaves is better in terms of throughput. However, the increase of the amount of relay nodes and the fact that these are more subject to environment interference (since these are considered as long links) make the throughput decrease significantly. Finally the throughput at the sink remains limited, compared to the outdoor scenario, for the reasons described previously in analysis of the Configuration A.

The third configuration-namely, Configuration $\mathrm{C}-\mathrm{is}$ shown in Figure 12c. In this configuration, the throughput at the leaves is significant. This could have been expected by taking into account the facts that (i) the links are shorts and, therefore, nearly not subject to interference; and (ii) the amount of concurrent transmissions remains limited. Since there are numerous relay nodes, the throughput at the relays is very low, because of the presence of multiple access collisions. Furthermore, the reflections off the environment reduce drastically the throughput at the sink when the relay probability of transmission increases. It can be observed that the value of $\tau_{\text {sink }}$ rapidly reaches a maximum for $q$ $\approx 0.05$, before decreasing rapidly for increasing values of the parameter $q$.

\section{Energy depletion rate}

First, regarding a BAN deployed in an outdoor environment, the energy consumption rate $\mathcal{E}$ is presented in Figure 13 as function of $q$ and for the three configurations of interest. It can be observed that the energy consumption rates of the three configurations present clearly different profiles. More precisely, Configuration A outperforms Configuration B which, in turn, is more energy efficient than Configuration C. Also, it can be observed that this remains true for any value of the node probability of transmission $q$.

The energy consumption rate in indoor scenarios is shown in Figure 14. It is noticeable that the values of the energy consumption in Figures 13 and 14 are approximately the same. Also, the relative performances 


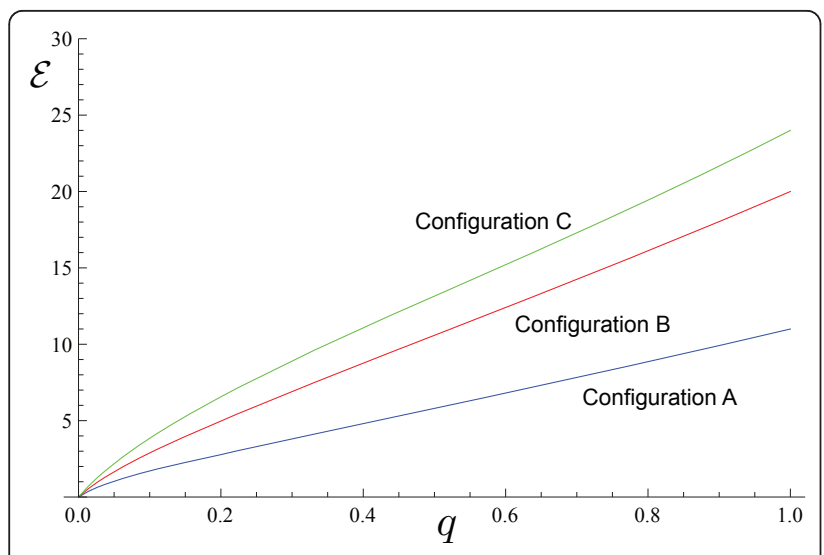

Figure 13 Energy consumption rate $\mathcal{E}$ of an outdoor hierarchical BAN with 16 nodes as a function of the leaves' probability of transmission and for different hierarchical configurations

of the three configurations in indoor scenarios are the same as in the outdoor case: Configuration A presents the lowest energy consumption rate, whereas Configuration $\mathrm{C}$ is the most energy consuming.

\section{E. An illustrative comparison with TDMA-based architectures}

In this article, we refer to the IEEE 802.15.6 standard and the slotted ALOHA MAC. Owing to its random access strategy, this MAC protocol could be of less interest if the traffic is high. In that case, a centralized, time division multiple access (TDMA) access might be more interesting and appealing. In this subsection, we provide the reader with an illustrative comparison between the slotted ALOHA and TDMA schemes, focusing on the delay exhibited by both strategies-in fact, the throughput of a TDMA scheme is equal to 1 .

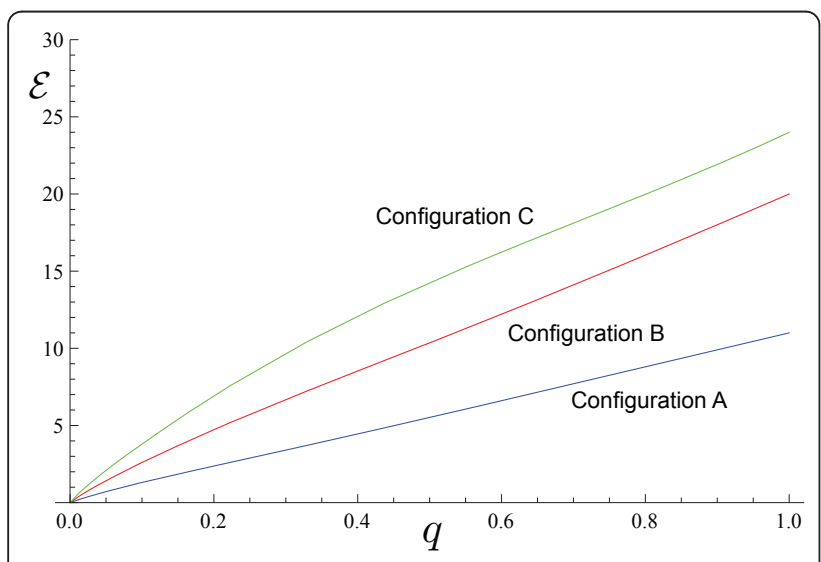

Figure 14 Energy consumption rate $\mathcal{E}$ of an indoor hierarchical BAN with 16 nodes as a function of the leaves' probability of transmission and for different hierarchical configurations.
In a slotted ALOHA system, the delay is directly related to the amount of (re-)transmissions needed to send (or forward) a packet. More precisely, the average number of transmissions can be written as

$$
\mathbb{E}[r]=\sum_{r=1}^{\infty} r Q_{r}
$$

where $Q_{r}$ is the probability of a successful communication realized after exactly $r$ transmissions (i.e., $r-1$ unsuccessful transmissions and a successful transmission). It can be expressed as

$$
Q_{r}=\left(1-P_{s}\right)^{r-1} P_{s} .
$$

In other words, $r$ is a geometric RV with parameter $P_{s}$, and one obtains

$$
\mathbb{E}[r]=\frac{1}{P_{S}},
$$

where the link probability of success $P_{s}$ depends on the scenario (i.e., outdoor or indoor, position of the nodes, etc.) and is given by relations (17), (18), and (19). Finally, the delay, expressed in number of time slots, is $D_{\mathrm{ALOHA}}=\mathbb{E}[r]=1 / P_{s}$.

In a TDMA system, the delay $D_{\text {TDMA }}$ depends on the amount of time to wait before a dedicated slot takes place. In a generic approach, it can be supposed that a relay node will allocate exactly one slot per sensor to receive its data and a slot per sensor for the forwarding uplink. In Figure 15, the slots' allocation (i.e., chronogram) is presented for each relay (noted $R_{1}, R_{2}, \ldots, R_{N_{2}}$ ). The time slots have a fixed duration of $T_{s}$ (dimension: [s]). As can be seen from Figure 15, each relay needs a frame of $N_{1}$ time slots to collect the (possibly generated) packets from its $N_{1}$ leaves. It then needs another frame ( $N_{1}$ time slots) to forward them to the sink. At this point, it needs to remain idle for $\left(N_{2}-2\right)$ frames, as the sink is busy collecting the packets from the other relays. This corresponds to assuming the same transmission rates at leaves and relay, and the same TDMA-based approach as at first and second layers. Therefore, the distance between two consecutive slots assigned to a given leaf is equal to $N_{1} \cdot N_{2}$ slots: when a leaf generates a packet, it needs to wait a number of slots between 0 (its slot is the current one) and $N_{1} \cdot N_{2}$ - 1 (its slot just passed). ${ }^{\mathrm{d}}$ As each number of slot has the same probability, the average delay (expressed in time slots) experienced by a given leaf node is

$$
\begin{aligned}
D_{\text {TDMA }} & =\frac{1}{N_{2} \cdot N_{1}-1} \sum_{i=0}^{N_{2} \cdot N_{1}-1} i \\
& =\frac{N_{2} \cdot N_{1}}{2} .
\end{aligned}
$$




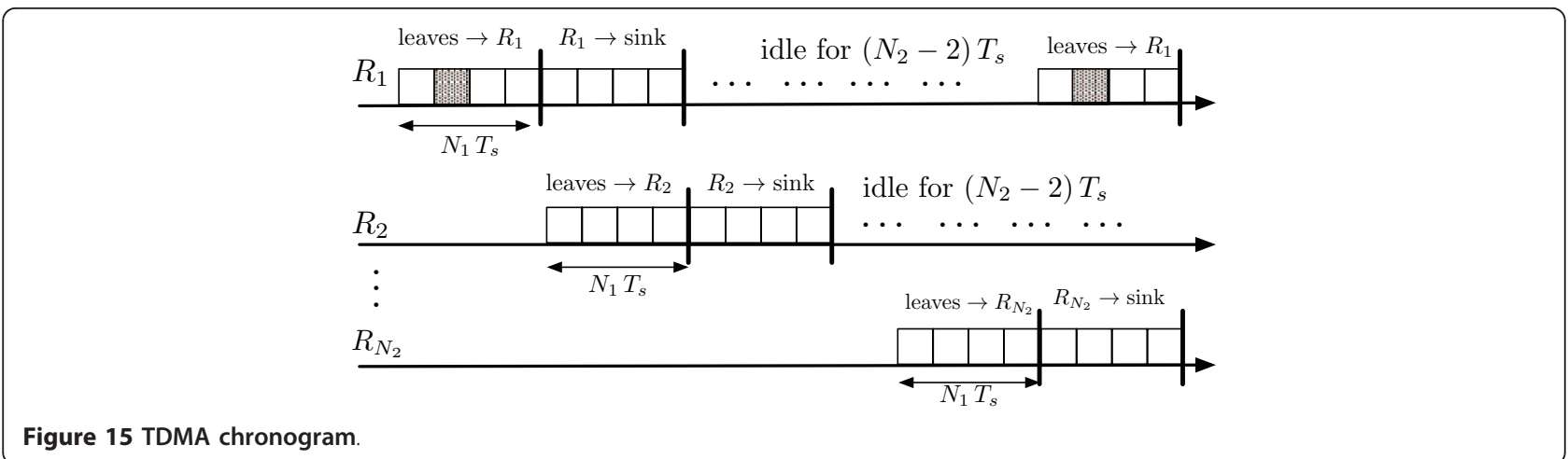

The above derivation for $D_{\text {TDMA }}$ represents an "average" scenario where a node generates at most a packet in an interval equal to $N_{1} \cdot N_{2}$ time slots: this corresponds to requiring that $\lambda \leq 1 /\left(N_{1} \cdot N_{2} \cdot T_{s}\right)$. In this case, expression (32) for the delay is correct. If, on the other hand, $\lambda>1 /\left(N_{1} \cdot N_{2} \cdot T_{s}\right)$, then it means that as soon as a leaf has transmitted a packet to its relay, it is likely to generate shortly a new packet, which will wait a period longer than (32). In general, it can be stated that

$$
\frac{N_{2} \cdot N_{1}}{2} \leq D_{\mathrm{TDMA}} \leq N_{2} \cdot N_{1} \text {. }
$$

In Figure 16, the average delay (expressed in contention time slots) incurred by a leaf to reach its relay is shown as a function of the transmission probability $q$. All curves refer to an indoor scenario. In the TDMA scheme, we consider the average expression ${ }^{e}$ (32). As expected, it can observed that, when the node probability of transmission is low, the slotted ALOHA significantly outperforms the TDMA scheme. However, for increasing probability of transmission, i.e., for increasing traffic load, there exists a critical threshold above which the TDMA scheme is to be preferred.

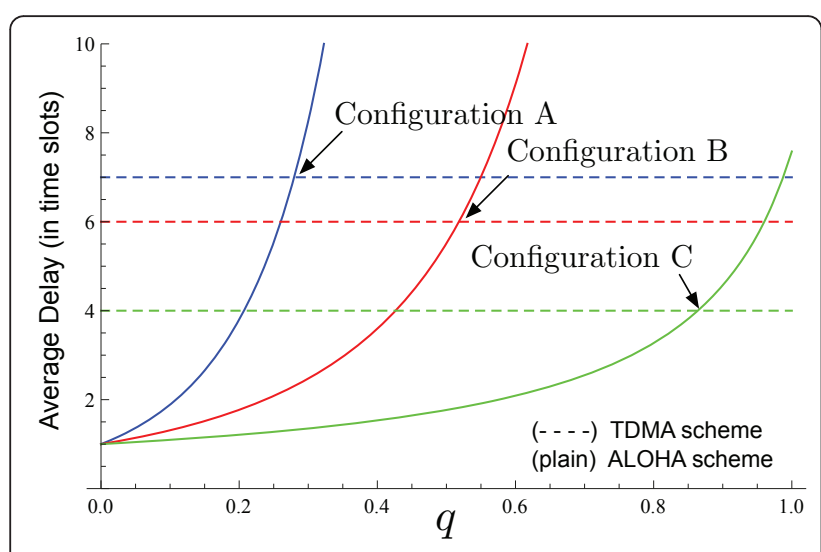

Figure 16 Average delay at the relay nodes for TDMA (expression (32)) and slotted ALOHA schemes as functions of sensor probability of transmission.
To summarize, as a TDMA-based scheme has a throughput $\tau=1$, it becomes very attractive for values of $q$ beyond the maximum of the considered slotted ALOHA system, as the latter becomes unstable, i.e., the value of the delay $D_{\mathrm{ALOHA}} \rightarrow \infty$ since $P_{s} \rightarrow 0$. In scenarios with low reporting rate, the slotted ALOHA scheme is to be preferred.

\section{Conclusions}

In this article, we have presented an analytic framework for the evaluation of the link probability of success in interference-limited BANs subject to fading. This analytic derivation is based on novel experimental measurements which highlight two characteristic propagation mechanisms in BANs deployed in indoor scenarios: onbody propagation, and propagation through reflections from the environment.

Regarding the impact of the topology, three configurations have been analyzed. These analyses showed significantly different performances, in terms of per-node throughput and energy consumption rate. It can be concluded that a decentralized topology in an outdoor environment presents the best tradeoff between the throughputs at the leaves, the relays, and the sink. Moreover, the shape of the throughput curves shows that it is very stable, i.e., any increase or decrease of the node generation rate does not significantly impact the per-node throughput, and it is the most efficient in terms of energy consumption rate. In an indoor environment, the balanced tree seems to be more suitable. Indeed, it presents a higher throughput for the sink, the relays, and the leaves at the same time.

In conclusion, multi-user BANs deployment and operation should take into account the specificities of environment and adapt the routing algorithms and clustering strategies accordingly. In the particular context of an indoor environment or when the traffic load is high, narrowband-shared spectrum techniques are not performant, and other MAC schemes, such as TDMA should be considered instead, even if they are more complex to implement. 


\section{Appendix}

The modeling of slow-scale fading as a log-normal distribution (that is, a zero-mean Gaussian in $\mathrm{dB}$ scale) raises mathematical difficulties, as shown in (12). The complementary cdf of a zero-mean log-normal RV is

$$
\zeta(z ; \sigma) \triangleq \frac{1}{2}+\frac{1}{2} \operatorname{erf}\left(\frac{-10 \log _{10} z}{\sigma \sqrt{2}}\right)
$$

where erf $\triangleq \frac{2}{\sqrt{\pi}} \int_{0}^{x} e^{-t^{2}} \mathrm{~d} t$ is the error function. The function $\zeta(z ; \sigma)$ is shown, in Figure 17, as a function of $z$ for $\sigma \in\{4,8,12,16\} \mathrm{dB}$. It can be observed that $\zeta(z ; \sigma)$ (i) saturates for $z \rightarrow \infty$, regardless of the value of $\rho$; and (ii) has the shape of a decreasing exponential function of $z$ (for a given value of $\sigma$ ). The $\zeta$ function can be approximated with a linear combination of negative exponential functions, as in $[45,46]$ :

$$
\zeta(z ; \sigma)=\sum_{m}^{\infty} c_{m} \exp \left(-a_{m} z\right) \approx \sum_{m}^{n} c_{m} \exp \left(-a_{m} z\right),
$$

where the coefficients $\left\{c_{m}\right\}_{m=1}^{n}$ and $\left\{a_{m}\right\}_{m=1}^{n}$ depend on $\sigma$ and can be determined in a least square sense by means of $q \geq 2 n$ known points of the $\zeta$ function. The Levenberg-Marquardt algorithm $[47,48]$ can be used to determine the coefficients $\left\{c_{m}\right\}$ and $\left\{a_{m}\right\}$ for different values of $\sigma$ and 10, 000 points over the interval $z \in[0$, $1,000]$. The corresponding values are reported in Table 1 along with the corresponding residual sum of squares.

\section{Endnotes}

${ }^{a}$ Note that, even though (3) holds for $d \geq d_{\text {ref }}, L_{0}$ can be intuitively interpreted as the (extrapolated) gain (adimensional, linear scale) at distance $d=0$. In other words, $L_{0}$ takes into account the loss due to antenna emission.

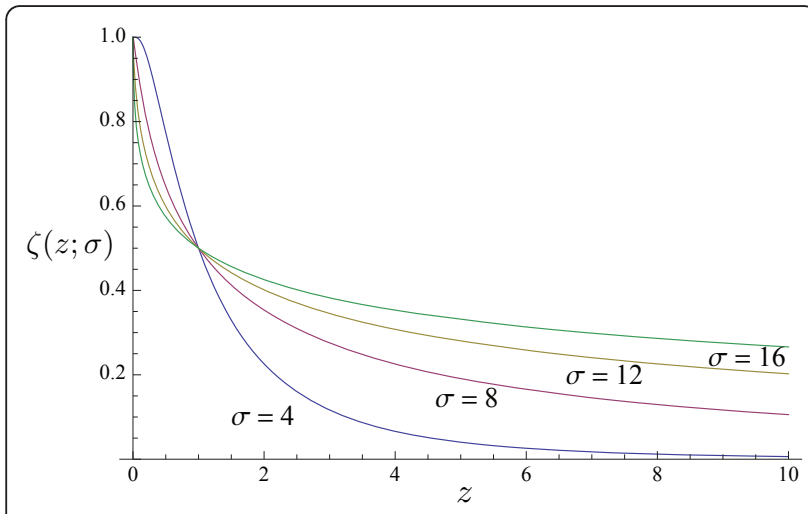

Figure 17 The function $\zeta(z ; \sigma)$ as a function of $z$, considering various values of $\sigma$ (in $\mathrm{dB}$ ).
Table 1 Coefficients for the approximation of the $\zeta$ function

\begin{tabular}{cccccccc}
\hline & $\boldsymbol{c}_{\mathbf{1}}$ & $\boldsymbol{a}_{\mathbf{1}}$ & $\boldsymbol{c}_{\mathbf{2}}$ & $\boldsymbol{a}_{\mathbf{2}}$ & $\boldsymbol{c}_{\mathbf{3}}$ & $\boldsymbol{a}_{\mathbf{3}}$ & Residual \\
\hline$\sigma=4$ & 0.49 & 0.75 & 0.49 & 0.75 & 0.03 & 0.16 & $4.68 \times 10^{-5}$ \\
$\sigma=6$ & 0.38 & 0.31 & 0.56 & 1.21 & 0.06 & 0.07 & $4.23 \times 10^{-6}$ \\
$\sigma=8$ & 0.59 & 1.32 & 0.34 & 0.18 & 0.06 & 0.02 & $1.04 \times 10^{-4}$ \\
$\sigma=10$ & 0.29 & 0.09 & 0.65 & 1.17 & 0.05 & 0.01 & $7.53 \times 10^{-4}$ \\
$\sigma=12$ & 0.04 & 0 & 0.24 & 0.04 & 0.70 & 0.93 & $3.52 \times 10^{-3}$ \\
$\sigma=14$ & 0.20 & 0.01 & 0.03 & 0 & 0.72 & 0.64 & $1.03 \times 10^{-2}$ \\
$\sigma=16$ & 0.18 & 0.01 & 0.70 & 0.49 & 0.04 & 0 & $1.67 \times 10^{-2}$ \\
\hline
\end{tabular}

${ }^{\mathrm{b}}$ Note that we use the $\log _{10}$ variant of the log-normal, since the widely used shadowing model uses an additive Gaussian variation expressed in $\mathrm{dB}$.

${ }^{\mathrm{c}}$ Note also that, with a slight abuse of notation, in (23) and (24), we indicate by $\zeta^{-1}(\cdot)$ the inverse of $\zeta(z ; \sigma)$ with respect to $\zeta$, with the implicit assumption that $\sigma$ is fixed.

${ }^{\mathrm{d}} \mathrm{We}$ assume that packet generation is at the beginning of a slot.

${ }^{\mathrm{e}}$ Note that this expression does not depend on $q$, as in TDMA systems, a leaf needs to wait for its assigned time slot.

\section{Acknowledgements}

This study is supported by the Belgian National Fund for Scientific Research (FRS-FNRS grants and FRIA grants).

\section{Author details}

'OPERA-Wireless Communications Group, Université Libre de Bruxelles, Belgium ${ }^{2}$ WASN Lab, Department of Information Engineering, University of Parma, Italy

\section{Competing interests}

The authors declare that they have no competing interests.

Received: 29 October 2010 Accepted: 7 October 2011 Published: 7 October 2011

\section{References}

1. J Xing, Y Zhu, A survey on body area network, in Proceedings of the 5th International Conference on Wireless Communications, Networking and Mobile Computing (WiCOM'09), Beijing, China, (2009)

2. E Monton, J Hernandez, J Blasco, T Herve, J Micallef, I Grech, A Brincat, V Traver, Body area network for wireless patient monitoring. IET Commun. 2(2), 215-222 (2008). doi:10.1049/iet-com:20070046

3. A Kailas, MA Ingram, Wireless communications technology in telehealth systems, in Proceedings of the IEEE Wireless VITAE Conference, Aalborg, Denmark, (May 2009)

4. D Bhatia, L Estevez, S Rao, Energy efficient contextual sensing for elderly care, in Proceedings of the 29th International Conference of the Engineering in Medicine and Biology Society (EMBS 2007), Lyon, France (August 2007)

5. J-M Dricot, S Van Roy, G Ferrari, F Horlin, P De Doncker, Link-level performance of indoor body area networks with centralized topologies. Open Electr Electron Eng J. 5, 9-18 (2011). doi:10.2174/ 1874129001105010009

6. J-M Dricot, G Ferrari, S Van Roy, F Horlin, Ph De Doncker, On the link layer performance of narrowband body area networks, in Proceedings of the Second International Conference on Emerging Network Intelligence (EMERGING 2010), Florence, Italy (October 2010)

7. E Reusens, W Joseph, G Vermeeren, L Martens, On-body measurements and characterization of wireless communication channel for arm and torso of human, in Proceedings of the 4th International Workshop on Wearable and 
Implantable Body Sensor Networks (BSN 2007), Aachen, Germany (March 2007)

8. JM Choi, H-J Kang, Y-S Choi, A study on the wireless body area network applications and channel models, in Proceedings of the 2008 Second International Conference on Future Generation Communication and Networking (FGCN '08), Hainan Island, China (2008)

9. N Katayama, K Takizawa, T Aoyagi, J-I Takada, H-B Li, R Kohno, Channel model on various frequency bands for wearable body area network. IEICE Trans Commun. E92.B(2), 418-424 (2009). doi:10.1587/transcom.E92.B.418

10. A Fort, C Desset, P De Doncker, P Wambacq, L Van Biesen, An ultrawideband body area propagation channel model-from statistics to implementation. IEEE Trans Microwave Theory Techn. 54(4), 1820-1826 (2006)

11. H Sawada, J Takada, S Choi, K Yazdandoost, R Kohno, Review of body area network channel model, in Proceedings of the IEICE General Conference, Meijo, Japan (2007)

12. J Ryckaert, PD Doncker, R Meys, A de Le Hoye, S Donnay, Channel model for wireless communication around human body. Electron Lett. 40(9), 543-544 (2004). doi:10.1049/el:20040386

13. S van Roy, C Oestges, F Horlin, P De Doncker, A comprehensive channel model for UWB multisensor multiantenna body area networks. IEEE Trans Antennas Propagation. 58(1), 163-170 (2010)

14. P Gupta, PR Kumar, The capacity of wireless networks. IEEE Trans Inf Theory 46(2), 388-404 (2000). doi:10.1109/18.825799

15. X Liu, M Haenggi, Throughput analysis of fading sensor networks with regular and random topologies. EURASIP J Wireless Commun Networks. 2005(4), 554-564 (2005)

16. C Guo, RV Prasad, M Jacobsson, Packet forwarding with minimum energy consumption in body area sensor networks, in Proceedings of the 7th IEEE Conference on Consumer Communications and Networking Conference (CCNC'10), Las Vegas, USA, 2010

17. L Yan, L Zhong, NK Jha, Energy comparison and optimization of wireless body-area network technologies, in Proceedings of the ICST 2nd International Conference on Body Area Networks (BodyNets '07), Florence, Italy (2007)

18. D Singh, H-J Lee, W-Y Chung, An energy consumption technique for global healthcare monitoring applications, in Proceedings of the 2nd International Conference on Interaction Sciences (ICIS '09), Seoul, Korea (2009)

19. N Abramson, The ALOHA system-another alternative for computer communications, in Proceedings Fall Joint Computer Conference, AFIPS Conference, Houston, USA (November 1970)

20. S Ullah, H Higgins, SMR Islam, P Khan, KS Kwak, On phy and mac performance in body sensor networks. EURASIP J Wireless Commun Networks. 2009, 1-7 (2009)

21. F Tobagi, Analysis of a two-hop centralized packet radio network-part l: slotted ALOHA. IEEE Trans Commun. 28(2), 196-207 (1980). doi:10.1109/ TCOM.1980.1094637

22. D Bertsekas, R Gallager, Data Networks, 2nd edn. (Prentice-Hall, Inc., Upper Saddle River, 1992)

23. R Nelson, L Kleinrock, The spatial capacity of a slotted ALOHA multihop packet radio network with capture. IEEE Trans Commun. 32(6), 684-694 (1984)

24. H Wu, Y Pan, Medium Access Control in Wireless Networks (Nova Science Pub Inc., Hauppauge, 2008)

25. A Jain, E Chang, Adaptive sampling for sensor networks, in Proceedings of the 1st International Workshop on Data Management for Sensor Networks, Toronto, Canada, 2004

26. S Stoa, I Balasingham, A decentralized mac layer protocol with periodic channel access for biomedical sensor networks, in First International Symposium on Applied Sciences in Biomedical and Communication technologies (ISABEL 2008), Aalborg, Denmark (2008)

27. Y Kwon, Y Fang, H Latchman, Performance analysis for a new medium access control protocol in wireless LANs. Wireless Networks. 10(5), 519-529 (2004)

28. J Weinmiller, M Schlager, A Festag, A Wolisz, Performance study of access control in wireless lans-IEEE 802.11 DFWMAC and ETSI RES 10 Hiperlan. Mobile Networks Appl. 2(1), 55-67 (1997). doi:10.1023/A:1013255927445

29. H Hsieh, R Sivakumar, IEEE 802.11 over multi-hop wireless networks: problems and new perspectives, in Proceedings of the IEEE Vehicular Technology Conference, 2002, Vancouver, Canada (September 2002)

30. EO Brigham, The Fast Fourier Transform and its Applications (Prentice-Hall, Englewood Cliffs, 1988)
31. S van Roy, C Oestges, F Horlin, P De Doncker, Propagation modeling for UWB body area networks: power decay and multi-sensor correlations, in Proceedings of the 10th IEEE International Symposium on Spread Spectrum Techniques and Application, Bologna, Italy (2008)

32. J ichi Takada, T Aoyagi, K Takizawa, N Katayama, H Sawada, T Kobayashi, KY Yazdandoost, $\mathrm{H}$ bang Li, R Kohno, Static propagation and channel models in body area, in COST 2100 6th Management Committee Meeting, TD(08)639 (2008)

33. A Goldsmith, Wireless Communications (Cambridge University Press, New York, 2005)

34. D Bertsekas, R Gallager, Data Networks (Prentice-Hall, Englewood Cliffs, 1991)

35. J-M Dricot, G Ferrari, S van Roy, F Horlin, P De Doncker, Outage, local throughput, and achievable transmission rate of body area networks, in Proceedings of the COST 2100 Meeting, no. TD(09) 939, Vienna, Austria (September 2009)

36. CE Jones, KM Sivalingam, P Agrawal, JC Chen, A survey of energy efficient network protocols for wireless networks. Wireless Networks. 7(4), 343-358 (2001). doi:10.1023/A:1016627727877

37. Y-A Le Borgne, J-M Dricot, G Bontempi, Principal component aggregation for energy efficient information extraction in wireless sensor networks, Knowledge Discovery from Sensor Data (Taylor and Francis, CRC Press, London, Boca Raton, 2007)

38. D Petrovic, R Shah, K Ramchandran, J Rabaey, Data funneling: routing with aggregation and compression for wireless sensor networks, in Proceedings of the 2003 IEEE International Workshop on Sensor Network Protocols and Applications, Anchorage, USA (2003)

39. S Madden, M Franklin, J Hellerstein, W Hong, TAG: a Tiny AGgregation Service for Ad-Hoc Sensor Networks, in Proceedings of the 5th ACM Symposium on Operating System Design and Implementation (OSDI), Boston, USA (2002)

40. E Jovanov, CCY Poon, G-Z Yang, YT Zhang, Body sensor networks: from theory to emerging applications. Trans Inf Tech Biomed. 13, 859-863 (2009)

41. G-Z Yang, Body Sensor Networks (Springer-Verlag New York, Inc., Secaucus, 2006)

42. X Liu, M Haenggi, Throughput analysis of random and regular networks. EURASIP J Wireless Commun Networking. 4, 554-564 (2005)

43. S Ahmed, MS Alam, Performance evaluation of important ad hoc network protocols. EURASIP J Wireless Commun Networking. 2006, 11 (2006). Article ID 78645

44. L Kleinrock, FA Tobagi, Carrier sense multiple-access modes and their throughput-delay characteristics. IEEE Trans Commun. 23, 1400-1416 (1983)

45. BG de Prony, Essai expérimental et analytique sur les lois de la dilatabilité des fluides élastique et sur celles de la force expansive de la vapeur de l'eau et de la vapeur de l'alkool, à différentes températures. J I'École Polytechnique 1(2), 24-76 (1795)

46. FG Lether, Elementary approximation for erf(x). J Quant Spectrosc Radiat Transfer. 49(5), 573-577 (1993). doi:10.1016/0022-4073(93)90068-S

47. K Levenberg, A method for the solution of certain non-linear problems in least squares. Quart Appl Math. 2, 164-168 (1944)

48. D Marquardt, An algorithm for least-squares estimation of nonlinear parameters. SIAM J Appl Math. 11, 431-441 (1963). doi:10.1137/0111030

doi:10.1186/1687-1499-2011-122

Cite this article as: Dricot et al:: Impact of the environment and the topology on the performance of hierarchical body area networks. EURASIP Journal on Wireless Communications and Networking 2011 2011:122. 